\title{
Bacterial encapsulins as orthogonal compartments for mammalian cell engineering
}

Felix Sigmund ${ }^{1,2,3}$, Christoph Massner ${ }^{1,2,3}$, Philipp Erdmann ${ }^{4}$, Anja Stelz1 ${ }^{1,2}$, Hannes Rolbieski ${ }^{1,2}$, Mitul Desai ${ }^{5}$, Sarah Bricault ${ }^{5}$, Tobias P. Wörner ${ }^{6}$, Joost Snijder 6,7 , Arie Geerlof ${ }^{8}$, Helmut Fuchs (10) 9 ,

Martin Hrabĕ de Angelis (10 ${ }^{9}$, Albert J.R. Heck (1) ${ }^{6}$, Alan Jasanoff ${ }^{5,10,11}$, Vasilis Ntziachristos ${ }^{1,12}$, Jürgen Plitzko ${ }^{4}$ \&

Gil G. Westmeyer (1,2,3

We genetically controlled compartmentalization in eukaryotic cells by heterologous expression of bacterial encapsulin shell and cargo proteins to engineer enclosed enzymatic reactions and size-constrained metal biomineralization. The shell protein (EncA) from Myxococcus xanthus auto-assembles into nanocompartments inside mammalian cells to which sets of native $(E n c B, C, D)$ and engineered cargo proteins self-target enabling localized bimolecular fluorescence and enzyme complementation. Encapsulation of the enzyme tyrosinase leads to the confinement of toxic melanin production for robust detection via multispectral optoacoustic tomography (MSOT). Co-expression of ferritin-like native cargo $(E n c B, C)$ results in efficient iron sequestration producing substantial contrast by magnetic resonance imaging (MRI) and allowing for magnetic cell sorting. The monodisperse, spherical, and iron-loading nanoshells are also excellent genetically encoded reporters for electron microscopy (EM). In general, eukaryotically expressed encapsulins enable cellular engineering of spatially confined multicomponent processes with versatile applications in multiscale molecular imaging, as well as intriguing implications for metabolic engineering and cellular therapy.

\footnotetext{
${ }^{1}$ Institute of Biological and Medical Imaging, Helmholtz Zentrum München, Ingolstädter Landstraße 1, Neuherberg 85764 , Germany. ${ }^{2}$ Institute of Developmental Genetics, Helmholtz Zentrum München, Ingolstädter Landstraße 1, Neuherberg 85764, Germany. ${ }^{3}$ Department of Nuclear Medicine, Technical University of Munich, Ismaninger Straße 22, Munich 81675, Germany. ${ }^{4}$ Department of Structural Biology, Max Planck Institute of Biochemistry, Am Klopferspitz 18, Martinsried 82152, Germany. ${ }^{5}$ Department of Biological Engineering, Massachusetts Institute of Technology, 77 Massachusetts Avenue, Cambridge 02139 Massachusetts, USA. ${ }^{6}$ Biomolecular Mass Spectrometry and Proteomics Group, Bijvoet Center for Biomolecular Research and Utrecht Institute for Pharmaceutical Sciences, Utrecht University, Padualaan 8, Utrecht 3584CH, The Netherlands. ${ }^{7}$ Snijder Bioscience, Spijkerstraat 114-4, Arnhem 6828 DN, The Netherlands. ${ }^{8}$ Institute of Structural Biology, Helmholtz Zentrum München, Ingolstädter Landstraße 1, Neuherberg 85764 , Germany.

${ }^{9}$ Institute of Experimental Genetics, Helmholtz Zentrum München, Ingolstädter Landstraße 1, Neuherberg 85764, Germany. ${ }^{10}$ Department of Brain \& Cognitive Sciences, Massachusetts Institute of Technology, 77 Massachusetts Avenue, Cambridge 02139 Massachusetts, USA. ${ }^{11}$ Department of Nuclear Science \& Engineering, Massachusetts Institute of Technology, 77 Massachusetts Avenue, Cambridge 02139 Massachusetts, USA. ${ }^{12}$ Chair for Biological Imaging, Technical University of Munich, Ismaninger Straße 22, Munich 81675, Germany. Correspondence and requests for materials should be addressed to G.G.W. (email: gil.westmeyer@tum.de)
} 
C ompartmentalization, the spatial separation of processes into closed subspaces, is an important principle that has evolved on several biological scales: multi-enzyme complexes that channel substrates, nanocompartments built entirely from proteins, as well as membrane-enclosed organelles, cells, and organs. Compartments make it possible to generate and maintain specific local conditions that can facilitate interactions and reactions in confined environments ${ }^{1}$, such that they can isolate toxic reaction products, protect labile intermediate products from degradation, or separate anabolic from catabolic processes $^{2}$. Whereas eukaryotes possess many membraneenclosed organelles, membranous compartments are not known in bacteria with a notable exception of magnetosomes in magnetotactic bacteria, in which specific reaction conditions are maintained which enable magnetic biomineralization ${ }^{3,4}$. However, nanocompartment shells built entirely from protein complexes can serve functions in prokaryotes that are analogous to eukaryotic organelles 5 .

Intense work has been invested in engineering compartments in prokaryotic systems and yeast to realize features such as substrate channeling for biotechnological production processes ${ }^{1,6,7}$. In contrast, no orthogonal compartments with self-targeting cargo molecules exist to date for use in mammalian cells. Such a system could, for instance, enable cellular engineering of reaction chambers that would endow genetically modified mammalian cells with new metabolic pathways that may include labile intermediate products or spatially confined toxic compounds. Engineered orthogonal compartments in eukaryotic cells may also enable size-constrained synthesis of biomaterials via, e.g., metal biomineralization processes occurring under specific localized environmental conditions.

With regards to protein complexes as building blocks for addressable nanocompartments, viruses and virus-like particles have been expressed in bacterial hosts to encase fluorescent proteins $^{8-12}$, enzymes ${ }^{13-16}$, and even multi-enzymatic processes $^{17,18}$. Similarly, bacterial microcompartments (BMC) such as Eut microcompartments and carboxysomes have been genetically engineered to load foreign cargo proteins such as fluorescent proteins ${ }^{19-21}$. In mammalian systems, vault proteins (vaults) have been explored, which are ribonucleoprotein complexes enclosed by $\sim 60 \mathrm{~nm}$ large envelope structures ${ }^{22}$ into which foreign cargo proteins such as fluorescent proteins or enzymes can be packaged ${ }^{23-25}$. However, vaults have openings on both ends and are endogenously expressed by many eukaryotic cells ${ }^{26}$. With respect to protein shell structures that can incorporate iron, the iron storage protein ferritin has been overexpressed to generate MRI contrast under certain conditions although its core size is only $\sim 6 \mathrm{~nm}$ containing only $\sim 2000$ iron atoms on average per core, which can result in only low magnetization ${ }^{27-29}$. Viral capsids such as the ones from cowpea chlorotic mottle virus (CCMV) have also been equipped with iron-binding sites that lead to accumulation of iron. Expression, assembly, and iron loading in mammalian cells, however, have not yet been demonstrated $^{30}$.

In search of a versatile nanocompartment-cargo system for heterologous expression in eukaryotic cells, we were intrigued by the recently discovered class of prokaryotic proteinaceous shell proteins called encapsulins because they possess a set of attractive features: (1) A single shell protein-without the need for proteolytic processing-is sufficient to form comparably large shelllike architectures $(\sim 18 \mathrm{~nm}$ or $\sim 32 \mathrm{~nm})$ auto-assembled from 60 , or 180 identical subunits with a triangulation number $(T)$ of one $(T=1)$ or three $(T=3)$, respectively ${ }^{31}$. (2) The assembled shells are $\mathrm{pH}$ resistant and temperature stable $\mathrm{j}^{32}$. (3) A versatile set of native cargo molecules including enzymes exist that are packaged into shell structures via specific encapsulation signals defined by a short terminal peptide sequence ${ }^{32}$. (4) The pore size of $\sim 5 \AA$ allows channeling small molecular substrates through the shell ${ }^{33}$. (5) Myxococcus xanthus (M. xanthus) encapsulin was also shown to posses cargo proteins $\mathrm{B}$ and $\mathrm{C}$, both containing rubrerythrin/ ferritin-like domains as well as highly conserved iron-binding ExxH motifs ${ }^{31}$ enabling import and sequestration of iron inside the nanoshell. Ferritin-like cargo proteins adopt an "open ferritin structure" and possess ferroxidase activity ${ }^{31,33}$. A model based on structural data from Thermotoga maritima encapsulins (with $T=$ 1) assumes that the ferritin-like protein docks into the shell where it obtains ferrous iron through the pores which it then oxidizes for deposition of up to an estimated 30,000 iron atoms per shell in the case of $M$. xanthus $31,33,34$. This amount is an order of magnitude more than can be contained inside a ferritin core expressed in eukaryotic cells. The function of the cargo protein $\mathrm{D}$, on the other hand, has so far not been understood. (6) The termini of the shell protein extend to the inner and outer surface, respectively, such that surface functionalizations are conveniently possible. The outer surface can, for instance, be functionalized to install specific targeting moieties ${ }^{35-37}$. Encapsulin variants were also purified when secreted from HEK293 cells to present glycosylated epitopes for an innovative vaccination approach ${ }^{38}$. Recently, the inner surface of the shell from T. maritima was also modified with silver-binding peptides to cause local silver precipitation in Escherichia coli, but the ferritin-like cargo was deleted to achieve this feature ${ }^{39}$. (7) Non-native cargo proteins including enzymes can be addressed to the inside of the nanocompartment via a short encapsulation signal ${ }^{11,40}$.

This excellent set of studies showed the feasibility and utility of biotechnological production of encapsulins as biomolecular scaffolds and targetable vehicles and probes.

We here introduce engineered encapsulins modified from M. xanthus in the context of genetic programming of orthogonal and addressable cellular compartments in mammalian cells. We demonstrate that eukaryotically expressed encapsulins not only auto-assemble at high density and without toxic effects but that self-targeting and encapsulation of cargo molecules still efficiently occur in mammalian cells. We furthermore show localized enzymatic reactions in the nanocompartment useful for optical and optoacoustic imaging, as well as confined iron accumulation within the nanocompartments that labels cells for detection by MRI. Importantly, we also show that encapsulins can serve as excellent gene reporters for electron microscopy due their spherical shape and their ability to load iron. These data demonstrate the value of encapsulins as genetic markers across modalities. In addition, the iron sequestration inside the nanoshells affords magnetic manipulation of cells genetically labeled with encapsulins.

\section{Results}

Encapsulin expression and self-assembly. Based on the favorable set of features introduced above, we chose to heterologously overexpress the encapsulin shell protein from $M$. xanthus in HEK293T cells. We tagged the nanoshell with an outward facing FLAG epitope $\left(\mathrm{A}^{\mathrm{FLAG}}\right)$ and found it to express strongly without and with the native cargo molecules from $M$. xanthus, denoted encapsulins $\mathrm{B}, \mathrm{C}$, and $\mathrm{D}^{31}$.

Co-expression of Myc-tagged B, C, or D alone, or a combination of all three non-tagged proteins (via co-transfection or a P2A construct, Fig. 1b), co-immunoprecipitated with $\mathrm{A}^{\text {FLAG }}$ as visualized on silver-stained SDS-PAGE (Fig. 1c, middle panel). A corresponding western blot against the FLAG (Fig. 1c, upper panel) or Myc-epitope (Fig. 1c, lower panel) confirmed the identities of the protein bands $\left(\mathrm{A}^{\mathrm{FLAG}}: 32.9 \mathrm{kDa},{ }^{\mathrm{Myc}} \mathrm{B}: 18.5 \mathrm{kDa}\right.$, ${ }^{\mathrm{Myc}} \mathrm{C}: 15.4 \mathrm{kDa},{ }^{\mathrm{Myc} D}$ : $\left.12.5 \mathrm{kDa}\right)$. 
a

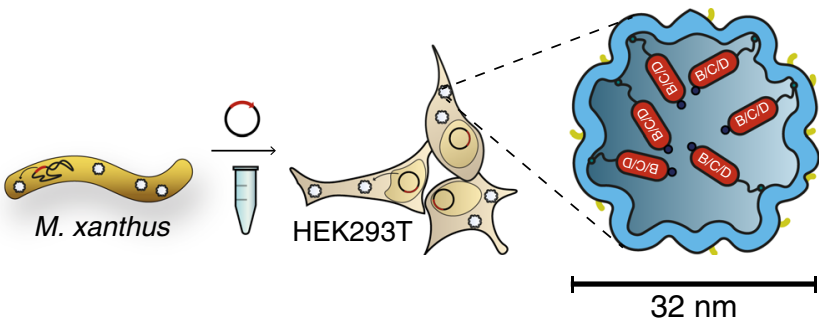

b

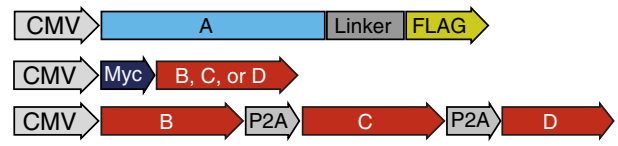

C
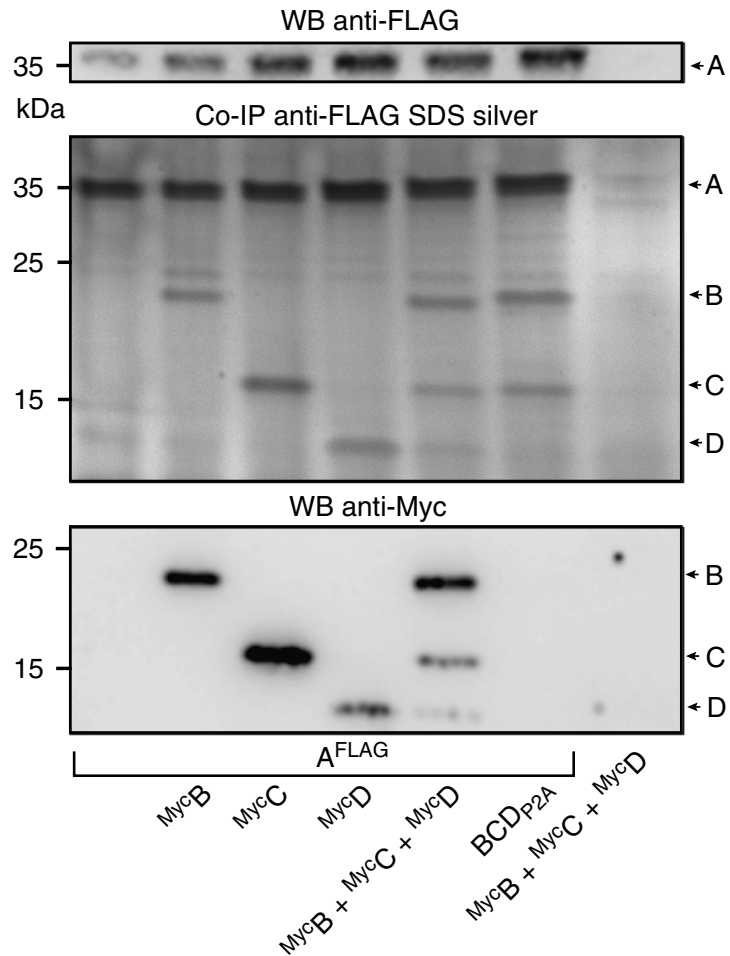

d

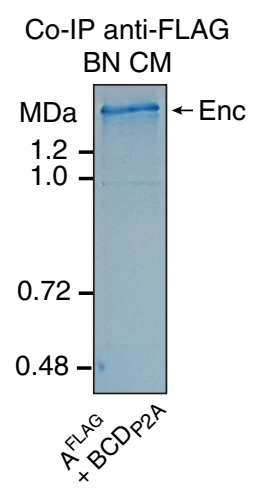

e

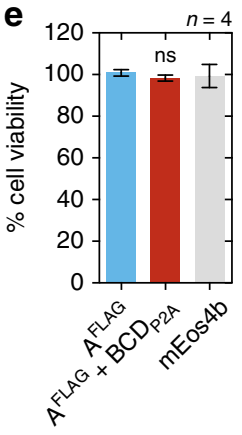

Fig. 1 Assembly of encapsulins and targeting of cargo in HEK293T cells. a Schematic of the heterologous expression of surface-modified encapsulin variants loaded with endogenous cargo proteins. b Genetic constructs encoding the shell protein A (light blue) with a FLAG-tag as C-terminal surface modification as well as individual Myc-tagged cargo proteins (red) B, C, and D that can also be combined in a multi-gene expression construct ( $B C D_{P 2 A}$ ). $\mathbf{c}$ Co-immunoprecipitation of $A^{F L A G}$ and silver-stained SDS-PAGE from cells co-expressing just $B, C$, or $D$, or a combination of these three proteins expressed either via a mixture of individual DNA constructs $\left({ }^{M y c} B+{ }^{M y c} C+{ }^{M y c} D\right)$, or by a multi-gene expression construct $\left(B C D_{P 2 A}\right)$. The top panel shows a western blot (WB) against the exterior FLAG-tag in $A^{F L A G}$. The bottom panel shows the corresponding WB against the Myc epitope. $\mathbf{d}$ Coomassie-stained Blue Native PAGE (BN CM) of purified material from HEK293T expressing $A^{\text {FLAG }}$ and BCD P2A yielding a band above 1.2 MDa. e Cell viability after $48 \mathrm{~h}$ of overexpression of encapsulins ( $A^{F L A G}$ ) with or without cargo $\left(B C D_{P 2 A}\right)$ assessed by an $L D H$ release assay. A construct expressing the fluorescent protein mEos4b served as a control. The bars represent the mean \pm SEM ( $p=0.1965$, Kruskal-Wallis, $n=4$; no significant (ns) differences at $\alpha=0.05$ were found in Dunn's multiple comparisons test between mEos $4 b$ and $A^{F L A G}$ expressed without or with BCDP2A)

Furthermore, a corresponding Blue Native PAGE (BN-PAGE) of immunoprecipitated FLAG-tagged material from cells expressing $\mathrm{A}^{\mathrm{FLAG}}$ together with $\mathrm{BCD}_{\mathrm{P} 2 \mathrm{~A}}$ revealed a band with an apparent molecular weight of above $1.2 \mathrm{MDa}$ indicating selfassembly of encapsulin protein complexes and self-targeting of all native cargo proteins (Fig. 1d).

The strong expression of $\mathrm{A}^{\mathrm{FLAG}}$ without or with loaded cargo did not result in a reduction of cell viability when compared to cells overexpressing a fluorescent protein as assessed by a viability assay based on lactate dehydrogenase (LDH) release (Fig. 1e).

We also generated a construct for a StrepTagII-labeled variant of the shell that co-expresses the ferritin-like Myc-tagged $\mathrm{C}$ as

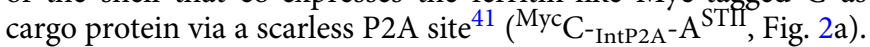

Material from HEK293T cells, conveniently purified via StrepTactin affinity chromatography, showed assembled nanospheres of $32.4 \pm 1.7 \mathrm{~nm}$ as the major component in single particle cryoelectron microscopy (cryo-EM) (Fig. 2b, Supplementary Fig. 1a, b), corresponding to the single band $>1.2 \mathrm{MDa}$ in size on $\mathrm{BN}$ PAGE (Fig. 2c, right panel). Again, no effect on cell viability was detected for this construct tested by a luciferase-based viability assay compared to $\mathrm{A}^{\mathrm{FLAG}}$ with and without cargo $\left(\mathrm{BCD}_{\mathrm{P} 2 \mathrm{~A}}\right)$, as well as controls without expression of encapsulins (EYFP and untransfected HEK293T) (Fig. 2d). Furthermore, N-terminal addition of the human BM40 (osteonectin SPARC) secretory signal peptide (SP) to the StrepTagII-modified encapsulin shell protein resulted in entry into the secretory pathway and robust 
a

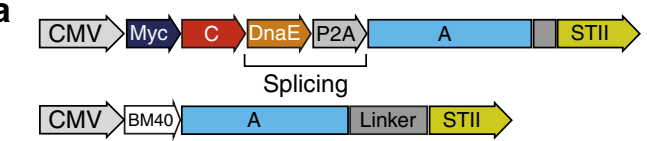

b

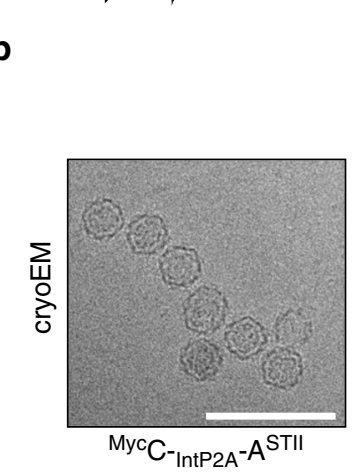

d

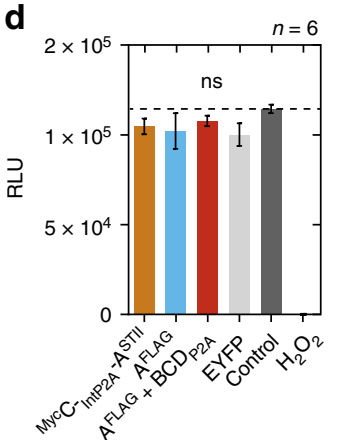

C

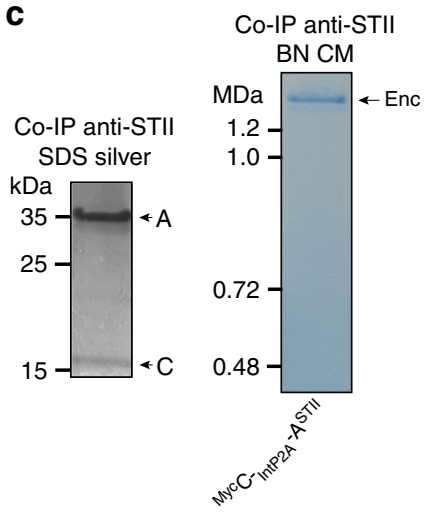

e

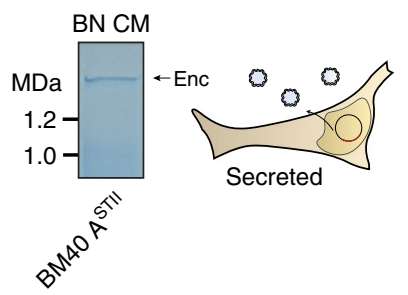

Fig. 2 Combined encapsulin:cargo construct and secreted encapsulin variant. a Scheme of a P2A bicistronic expression construct encoding StrepTagll-tagged ${ }^{\left({ }^{S T I}\right)}$ nanocompartments containing Myc-tagged $C$ as cargo protein $\left({ }^{\mathrm{Myc} C}{ }_{-}{ }_{\text {IntP2A }}-\mathrm{A}^{\mathrm{STII}}\right)$ as well as a variant with an $\mathrm{N}$-terminal BM40 secretion peptide and StrepTagII ${ }^{(\mathrm{STII})}$. b Cryo-electron microscopy image of material from HEK293T cells expressing ${ }^{\mathrm{Myc} C}{ }^{-}{ }_{\text {IntP2A }}-\mathrm{A}^{\mathrm{STIl}}$ purified via Strep-tag II/Strep-Tactin XT affinity chromatography showed the assembled nanospheres of $\sim 32 \mathrm{~nm}$ diameter. Scale bar is $100 \mathrm{~nm}$. c The corresponding BN-PAGE analysis of the identical material revealed a single band larger than 1.2 MDa. The accompanying silver-stained SDS-PAGE showed the coprecipitation of the cargo ${ }^{\mathrm{Myc}} \mathrm{C}$ with the StrepTagll-modified nanoshell. d Luciferase-based cell viability assay after $48 \mathrm{~h}$ of overexpression of ${ }^{\mathrm{Myc}} \mathrm{C}_{-\mathrm{IntP2A}} \mathrm{A}^{\mathrm{STII}}$ and $\mathrm{A}^{\mathrm{FLAG}}$ with or without cargo $B C D_{\text {P2A. }}$. Cells overexpressing the fluorescent protein EYFP as well as untransfected HEK293T cells served as negative controls. To induce toxicity as positive control, untransfected HEK293T cells were treated with $1 \mathrm{mM} \mathrm{H}_{2} \mathrm{O}_{2} 24 \mathrm{~h}$ prior to the assay. The bars represent the mean \pm SEM ( $p$ $=0.442$ excluding the positive control, Kruskal-Wallis, $n=6$; no significant (ns) differences at $\alpha=0.05$ were found in Dunn's multiple comparisons test between any of the encapsulin:cargo conditions and either EYFP or Control). e BN CM loaded with cell culture supernatant of HEK293T cells expressing $\mathrm{A}^{\mathrm{STII}}$ with an $\mathrm{N}$-terminal $\mathrm{BM} 40$ secretion signal showed a single band $>1.2 \mathrm{MDa}$

secretion of StrepTagII-modified encapsulins from HEK293T cells as shown by Coomassie-stained BN-PAGE of material present in the cell culture supernatant (Fig. 2e).

In vivo expression of encapsulins. To achieve in vivo expression of encapsulins, we generated a coexpression construct that encoded both the nanoshell $A^{\mathrm{FLAG}}$ and the ferritin-like protein $B$ from a single plasmid that was small enough to be packaged into an Adeno-associated virus (AAV) (Supplementary Fig. 1c). After

transduction of murine brains via intracranial injections of this viral vector co-expressing $\mathrm{A}^{\mathrm{FLAG}}$ and $\mathrm{B}^{\mathrm{M} 7}$ by a P2A peptide, we observed robust neuronal expression of the shell protein (Supplementary Fig. 1f, i). Silver-stained BN-PAGE and SDS-PAGE of immunoprecipitated (anti-FLAG) proteins extracted from murine brain showed that the nanocompartments assembled in vivo and that the cargo $\mathrm{B}^{\mathrm{M} 7}$ was associated with the shell (Supplementary Fig. 1g, j). Similar in vivo results could be obtained by $\mathrm{co}^{-}$ expressing the nanoshell and ferritin-like B cargo via an IRES site (Supplementary Fig. 1h).

Encapsulation of engineered cargo. We next tested whether non-natural cargo molecules could be efficiently targeted into the nanocompartments. We thus C-terminally appended a minimal encapsulation signal, which we found to only necessitate eight amino acids (EncSig), to the photoactivatable fluorescent protein mEos $4 b^{42}$, coexpressed it with $A^{\text {FLAG }}$ and found by coimmunoprecipitation and BN-PAGE analysis under an UV imager that the cargo readily associated with the encapsulin shell (Fig. 3b).

Selective degradation of non-encapsulated cargo proteins. Importantly, we could also selectively enrich cargo proteins to the encapsulin lumen by fusing an FKBP12-derived destabilizing domain (DD) that labels the cargo for rapid degradation unless it is shielded from proteasomal machinery ${ }^{43}$. We show that coexpressing $\mathrm{A}^{\mathrm{FLAG}}$ and DD-mEos4b-EncSig in HEK293T yielded significantly higher mean fluorescence values than DD-mEos4bEncSig alone, indicating that cargos inside the encapsulin are protected from proteolytic degradation (Fig. 3c, d). Confocal microscopy revealed that coexpression of DD-mEos $4 \mathrm{~b}-\mathrm{EncSig}$ with $\mathrm{A}^{\mathrm{FLAG}}$ shows green fluorescence throughout the cytosol but not in the nucleus, whereas the absence of the encapsulin shell ablated the fluorescence signal. In a positive control in which DDmEos $4 \mathrm{~b}$-EncSig was stabilized by adding a small molecule instead of encapsulating it, fluorescence was observed throughout the cell including the nucleus (Fig. 3e).

We then purified encapsulins co-expressed with and without DD-mEos4b-EncSig as cargo to determine their native mass and found that in the absence of cargo, also smaller nanospheres assembled consistent with the known configuration as 60-mers with $T=1$ symmetry (Supplementary Fig. 2 ). We estimated that on average $\sim 60$ fluorescent proteins per nanoshell were enclosed as confirmed by gel densitometry (Supplementary Fig. 3a, b). We furthermore found that the FLAG-tagged encapsulin shell is phosphorylated (Supplementary Fig. 3c-e).

Simultaneous encapsulation of sets of engineered cargo. We next wanted to assess whether multiple engineered cargo molecules could be encapsulated together. We thus fused the two halves of split PAmCherry1 (PA-s1, PA-s2) to either B or C (B-PA-s2: $27.0 \mathrm{kDa}, \mathrm{C}-\mathrm{PA}-\mathrm{s} 1: 33.1 \mathrm{kDa}$ ) and tested for bimolecular fluorescence complementation (BiFC) within the nanocompartment $^{44}$ (Fig. 4a, b). Either of these components could be co-immunoprecipitated with $\mathrm{A}^{\mathrm{FLAG}}$ as shown by silver-stained SDS-PAGE (Fig. 4b, Supplementary Fig. 4a). The photoactivation of the complemented split PAmCherryl inside the encapsulins could also be detected via fluorescence imaging of the corresponding BN-PAGE (Fig. 4b, right panel, Supplementary Fig. 4a). Co-expression of both split halves together with $\mathrm{A}^{\mathrm{FLAG}}$ lead to a strong increase of photoactivatable fluorescent signal throughout the cytosol of HEK293T cells as quantified by confocal microscopy compared to cells that did not express $\mathrm{A}^{\mathrm{FLAG}}$ (Fig. 4c). 
a

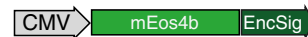

CMV $>D$
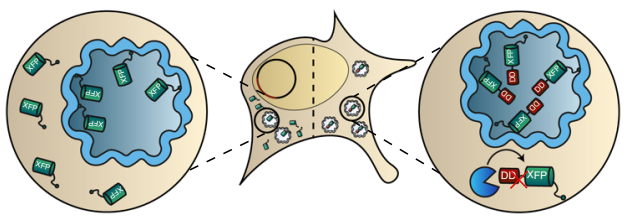

e

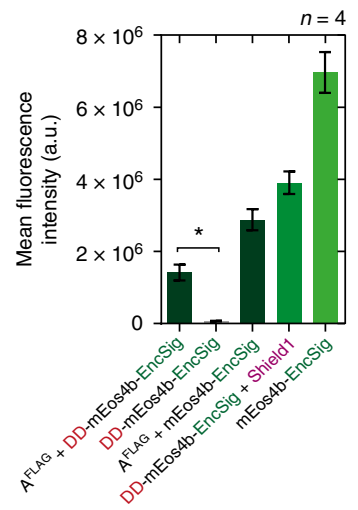

b

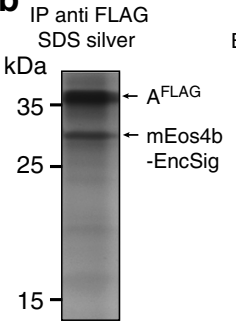

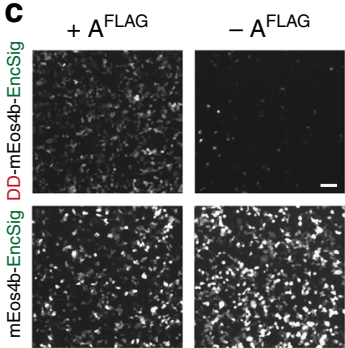

BN Fluor.

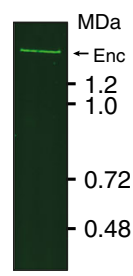

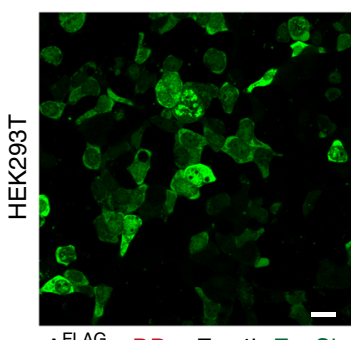

$A^{F L A G}+D D-m E o s 4 b-E n c S i g$

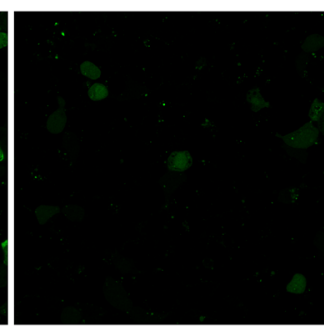

DD-mEos4b-EncSig

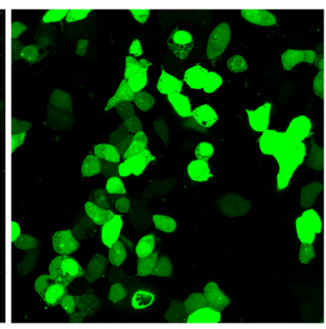

DD-mEos4b-EncSig + Shield1

Fig. 3 Selective degradation of non-encapsulated cargo. a Schematic of genetic construct showing a minimal C-terminal encapsulation signal (EncSig) fused to the photoactivatable fluorescent protein mEos $4 b$ (mEos4b-EncSig) to associate it to the inner surface of the nanocompartment. When mEos $4 b$-EncSig is $\mathrm{N}$-terminally fused to an FKBP12-derived destabilizing domain (DD), it is degraded by the proteasome unless it is sequestered into the encapsulin shell. This strategy thus selectively enriches cargo inside the lumen of the nanocompartment. $\mathbf{b}$ Cargo loading of mEos4b-EncSig into the nanocompartment composed of $A^{F L A G}$ was demonstrated by co-immunoprecipitation (Co-IP) against the FLAG epitope followed by silver-stained SDS-PAGE (left panel). Corresponding analysis of whole cell lysate by BN-PAGE on a UV imager shows fluorescence of the native encapsulin band indicating the presence of the mEos4b-EncSig cargo. c Representative $2 \times 2$ table of epifluorescence microscopy images from HEK293T cells co-expressing DD-mEos4b-EncSig with or without $A^{F L A G}$ (upper row) compared to coexpression of the cargo without destabilizing domain (mEos4b-EncSig, lower row). Scale bar represents $50 \mu \mathrm{m}$. d Corresponding quantification of the fluorescence intensities as exemplified in c. Co-expression of $A^{\mathrm{FLAG}}$ significantly protects degradation of DDmEos4b-EncSig ( $p=0.0286$, Mann-Whitney test, $n=4$ biological replicates, error bars represent mean \pm SEM). DD-mEos4b-EncSig can also be stabilized by adding the small molecule Shield1 $(0.5 \mathrm{mM}$, magenta label) to the cell culture medium. e Confocal microscopy images of HEK293T expressing DDmEos4b-EncSig with or without $A^{F L A G}$. As a reference DD-mEos4b-EncSig was stabilized via the addition of 0.5 mM Shield1. Please note that the contrast of all images was linearly adjusted to the same extent optimizing for the condition shown on the left, which resulted in partial oversaturation of the condition shown on the right. Scale bar represents $20 \mu \mathrm{m}$

Compartmentalized enzymatic reactions. To showcase the use of the eukaryotically expressed encapsulins as bioengineered reaction chambers with pores that can constrain passage of reactants and reaction products, we targeted several enzymes to the nanocapsules. In the presence of $\mathrm{A}^{\mathrm{FLAG}}$, the split luciferase ${ }^{45}$ parts LgBit and SmBit fused to C and B (C-LgBit: $32.7 \mathrm{kDa}, \mathrm{B}-$ SmBit: $19.6 \mathrm{kDa}$ ) were complemented to functional enzymes as demonstrated by bioluminescence detection from BN-PAGE (Fig. 4d, left) and from total lysate (Fig. 4d, bar graph on the right). Importantly, only very low luminescence signals were detected when the encapsulin shell was not present indicating that using split protein approaches can also ensure confined enzyme activity inside the capsules, in addition to the strategy for selective enrichment of cargo inside the nanocompartment as shown in Fig. 3.

Bioengineered melanosomes as gene reporters for MSOT. We subsequently sought to utilize selective passage of small substrates through the nanoshell to load the compartments with tyrosinase as cargo which is the sole enzyme generating the photoabsorbing polymer melanin from the amino acid tyrosine. Because of these attractive features, tyrosinase has been used as a gene reporter for optoacoustic tomography ${ }^{46,47}$, an imaging modality that maps the distribution of photoabsorbing molecules in tissue by locating the ultrasonic waves that they emit in response to local heating upon laser absorption ${ }^{48,49}$. However, melanin production is toxic to cells if not confined in melanosomes, which are membranous compartments of specialized cells ${ }^{50,51}$. We thus chose a soluble tyrosinase from Bacillus megaterium ${ }^{52}$ that we thought could still be functional as a fusion protein to the native cargo $\mathrm{D}\left({ }^{\mathrm{Myc}} \mathrm{D}\right.$ BmTyr: $47.7 \mathrm{kDa}$ ) serving as targeting moiety (Fig. 5a). Indeed we could observe generation of melanin on the BN-PAGE band corresponding to the assembled nanocompartment (Fig. 5b). In cells expressing the encapsulin-targeted tyrosinase and the shell $\mathrm{A}^{\mathrm{STII}}$, we observed robust melanin formation by bright-field microscopy without the strong toxicity apparent in the morphology of control cells expressing just the tyrosinase (Fig. 5c, white arrows). Encapsulation of the tyrosinase also led to a significant increase in cell viability as assessed by a luciferase-based viability assay (Fig. 5d). Cells expressing melanin-producing encapsulins were dark in color (Fig. 5e, inset) and thus generated intense photoacoustic signal even when referenced against strongly absorbing synthetic ink with an optical density of 0.2 (Fig. 5e).

Similarly, we showed that the engineered peroxidase APEX2 ${ }^{53}$ can polymerize Diaminobenzidine (DAB) when targeted to the nanocompartment (APEX2-EncSig; $31.0 \mathrm{kDa}$ ) as indicated by the generation of photoabsorbing $\mathrm{DAB}$ polymers associated with the BN-PAGE band corresponding to the assembled nanosphere (Supplementary Fig. 4b). 
a

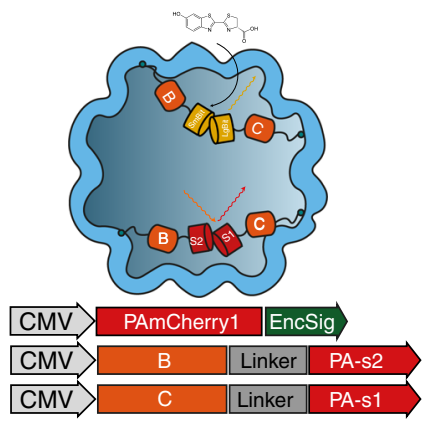

C

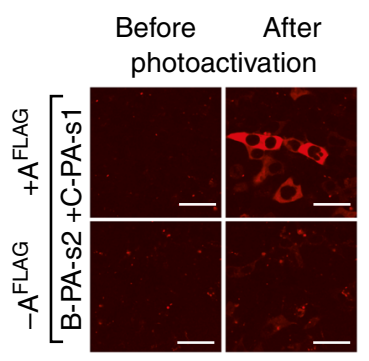

b

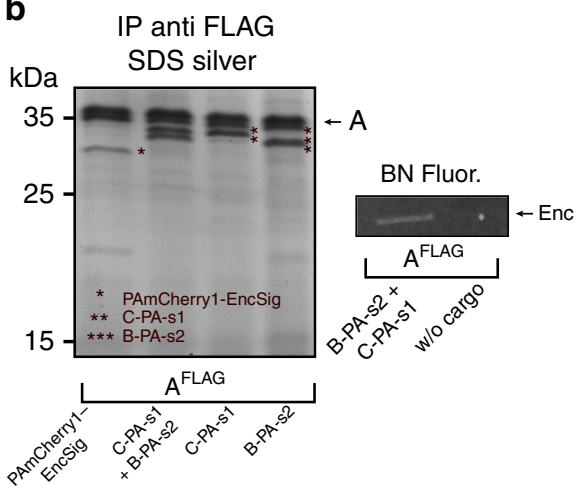

d

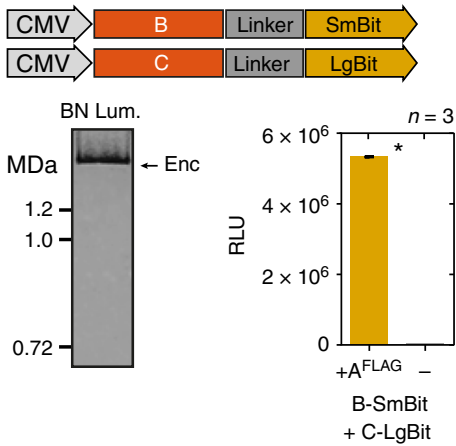

Fig. 4 Multi-component processes and enzymatic reactions can be targeted to encapsulins in mammalian cells. a Overview schematic of sets of cargo molecules for bimolecular fluorescence and enzyme complementation inside the nanocompartment. Targeting of foreign cargo proteins can be achieved either via a minimal C-terminal encapsulation signal (EncSig) or via C-terminal fusions to the native cargo proteins B, C, or D. b Silver-stained SDS-PAGE from a co-immunoprecipitation ( $\mathrm{Co}-\mathrm{IP}$ ) of $\mathrm{A}^{\mathrm{FLAG}}$ co-expressed with photoactivatable mCherry1 with EncSig (PAmCherry1-EncSig) or with either one of the halves of split PAmCherry1 fused to $C$ or $B$, or a combination of both (C-PA-s1 + B-PA-s2). Fluorescence originating from complemented split PAmCherry1 inside the encapsulins was detected on BN-PAGE loaded with whole cell lysates of cells expressing $A^{F L A G}$ and C-PA-s1 + B-PA-s2 after 2 min of photoactivation (PA) on an UV imager. c Live cell confocal microscopy images (scale bar represents $20 \mu \mathrm{m}$ ) of HEK293T cells expressing B-PAs1 and CPAs2 with or without the shell-protein A FLAG before and after $60 \mathrm{~s}$ of photoactivation (PA) with $405 \mathrm{~nm}$ (upper panel) demonstrating efficient bimolecular fluorescence complementation inside encapsulin compartments. Fluorescence of photoactivated split PAmCherry1 was excited using a $561 \mathrm{~nm}$ laser. Fluorescence signals of the sample without and with $A^{\text {FLAG }}$ were quantified by calculating the ratio of the mean signal after PA divided by the signal before PA. The bars in the lower panel represent the mean fluorescence intensity ratios averaged over independent transfection experiments \pm SEM $(p=0.0123$, unpaired $t$-test, $n=3$ ). d Luminescence signal from BN-PAGE incubated with luciferase substrate and loaded with whole cell lysates of HEK293T coexpressing split luciferase fragments fused to either B or C (B-SmBit, C-LgBit) and $A^{F L A G}$ (left panel). The luminescent band corresponds to the complemented split luciferase inside the assembled nanocompartment. The bar graph (right panel) shows the corresponding total luminescence signals from the cell lysates expressing B-SmBit and C-LgBit with or without $A^{F L A G}$, (mean \pm SEM across three independent transfection experiments, in each experiment three technical replicates were averaged, $p<0.0001$, unpaired $t$-test, $n=3$ )

Since the electrophoretic mobility of protein complexes on BNPAGE also depends on their hydrodynamic size and shape ${ }^{54}$, cargo-loading could be confirmed by observing an identical migration behavior of loaded as compared to unloaded capsules (Supplementary Fig. 4c).

Moreover, we targeted the putative cystathionine $\gamma$-lyase (SmCSE) to the nanospheres via an EncSig (smCSE-EncSig: $43.9 \mathrm{kDa}$ ) as shown by Co-IP with $\mathrm{A}^{\mathrm{FLA}}$ (Supplementary Fig. $4 \mathrm{~d})$. In the presence of L-cysteine, this enzyme was reported to catalyze a conversion of cadmium acetate in aqueous solution into cadmium sulfide (CdS) nanocrystals such that they would generate a photoluminescence signal under UV illumination characteristic for crystal formation at quantum confined sizes ${ }^{55}$. Indeed, we could detect a photoluminescence signal from the BN-PAGE band corresponding to encapsulin loaded with SmCSE-EncSig after on-gel incubation with cadmium acetate and L-cysteine indicating that the smCSE-EncSig cargo was enzymatically active when bound into the shell (Supplementary Fig. 4d).
Size-constrained iron biomineralization. Another reason to choose encapsulins from M. xanthus was that it was previously reported to deposit iron via the ferritin-like cargo B and C into relatively large compartments $(\sim 32 \mathrm{~nm}, T=3)^{31}$. We thus investigated whether this functionality could also be realized in eukaryotic cells to enable spatially confined iron deposition sequestered away from the complex signaling network controlling mammalian iron homeostasis. We thus generated a stable cell line co-expressing the nanoshell $\left(\mathrm{A}^{\mathrm{FLAG}}\right)$ with all native cargo proteins $(B, C, D)$ via a dual-promoter construct (Fig. 6a). In this cell line, we observed long-term and robust expression of all components shown by co-immunoprecipitation with $\mathrm{A}^{\mathrm{FLAG}}$ and by immunocytochemistry against the external FLAG-epitope (Fig. 6b, left panel, Supplementary Fig. 5a). Transient co-expression of the ferrous iron transporter MmZip14 ${ }^{\mathrm{FLAG}}$ (Zip14) in the stable cell line resulted in a robust dose-dependent iron loading (with ferrous ammonium sulfate (FAS) at concentrations between $0.25-1.25 \mathrm{mM}$ ) already after $48 \mathrm{~h}$ of supplementation as detected on BN-PAGE via 
a
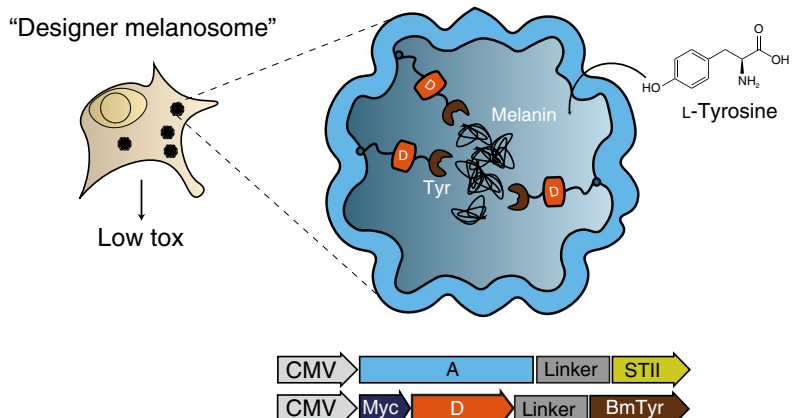

C

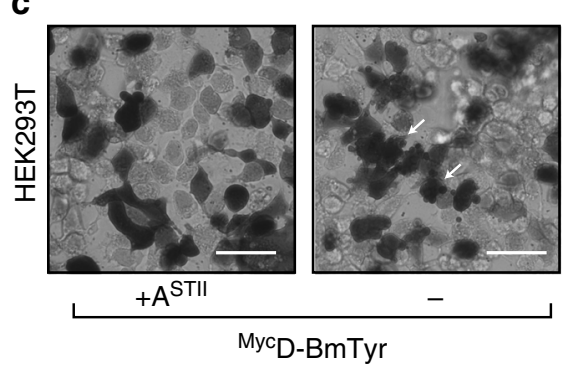

d

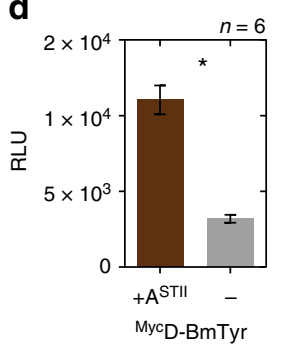

b
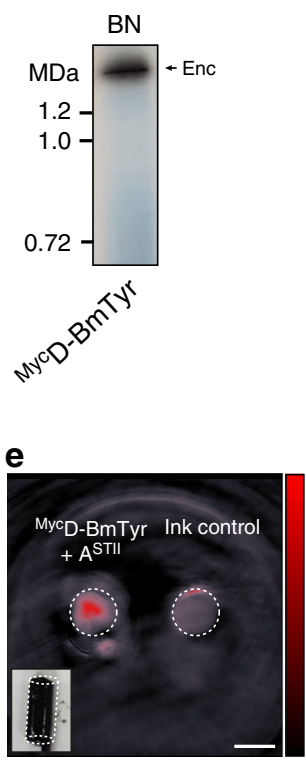

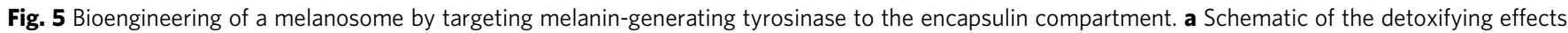
of compartmentalized melanin production by encapsulated tyrosinase from Bacillus megaterium targeted to the nanocompartment via fusion to the native cargo D. The substrate L-tyrosine enters the compartment via the pores in the nanoshell. b BN-PAGE showing on-gel production of melanin via tyrosinase expressed in HEK293T cells fused to Myc-tagged encapsulin-cargo D (MycD-BmTyr) to encapsulate it in the assembled nanoshell. Dark colorization of the band was observed after incubation with $2 \mathrm{mM}$ L-tyrosine and $100 \mu \mathrm{M} \mathrm{CuCl}{ }_{2}$ in PBS (pH 7.4 ) for $1 \mathrm{~h}$ at $37{ }^{\circ} \mathrm{C}$. c Bright-field images of $\mathrm{HEK} 293 \mathrm{~T}$ cells expressing MycD-BmTyr with and without StrepTagll-modified shell ( ${ }^{\mathrm{STII}}$ ) after $48 \mathrm{~h}$ of expression. Twenty four hours post transfection, cells were supplemented with $1 \mathrm{mM}$ L-tyrosine and $10 \mu \mathrm{M} \mathrm{CuCl}_{2}$. Cell protrusions (white arrows) were apparent indicating toxic effects of overexpression of nonencapsulated tyrosinase. Scale bar: $20 \mu \mathrm{m}$. d Corresponding luciferase-based viability assay of HEK293T cells treated as in c overexpressing Myc D-BmTyr with or without $A^{S T I I}$ after $48 \mathrm{~h}$. (The bars represent the mean $\pm \mathrm{SEM}, n=6, p<0.0001$, unpaired $t$-test.) e Images of two tubular phantoms (transversal slice) obtained by multispectral optoacoustic tomography (MSOT). The phantoms were filled with $\sim 10^{7}$ cells in $1.5 \%$ low melting agar expressing Myc ${ }^{2}$ BmTyr with $\mathrm{A}^{\mathrm{STII}}$ (supplementation as in $\mathbf{c}$ and $\mathbf{d}$ ) or containing highly concentrated ink $(\mathrm{OD}=0.2)$ as control showing the intense contrast obtained

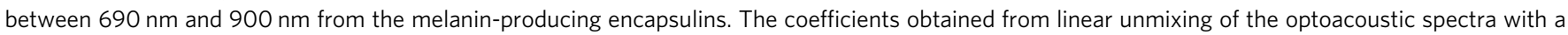
melanin reference spectrum are displayed on the red colormap overlaid on the image obtained at $720 \mathrm{~nm}$. The lower left inset shows a color photograph of the tubular phantom containing the cells. Scale bar: $3 \mathrm{~mm}$

DAB-enhanced Prussian Blue staining (DAB PB) (Fig. 6b, right panel).

Efficient iron loading could also be achieved by transient expression of $\mathrm{A}^{\mathrm{FLAG}}+\mathrm{BCD}_{\mathrm{P} 2 \mathrm{~A}}$ together with Zip14. Under these conditions, iron supplementation with $\sim 0-3 \mathrm{mM}$ FAS for $48 \mathrm{~h}$ led to a substantial dose-dependent iron loading of the nanocompartment that saturated at $\sim 1 \mathrm{mM}$ FAS as shown by Coomassie and DAB-enhanced Prussian Blue BN PAGE (Fig. 6c, upper panel, Supplementary Fig. 5b). Interestingly, when we tested the cargo molecules individually for their ability to load iron into the nanosphere, we found that co-expression of only $\mathrm{B}$ or $\mathrm{C}$ generated equally intense $\mathrm{DAB} P B$ bands as compared to $\mathrm{BCD}_{\mathrm{P} 2 \mathrm{~A}}$, indicating that either $\mathrm{B}$ or $\mathrm{C}$ is sufficient for iron deposition inside the nanocompartment.

In contrast, co-expression of D with $\mathrm{A}^{\mathrm{FLAG}}$ or any of the cargo molecules without the presence of $A^{\text {FLAG }}$ did not lead to discernable DAB PB signals (Fig. 6c, lower panel, Supplementary Fig. 5c). In a standard cell viability assay, we found no impairment of the cells when Zip14 was co-expressed together with $\mathrm{A}^{\mathrm{FLAG}}$ and $\mathrm{BCD}_{\mathrm{P} 2 \mathrm{~A}}$ or just $\mathrm{B}$. However, $7 \%$ of cells showed reduced viability when the cargos $\mathrm{BCD}_{\mathrm{P} 2 \mathrm{~A}}$ were expressed without the nanocompartment $(p=0.0238$, Mann Whitney test, $n=3$ ) or when only the fluorescent protein mEos4b-EncSig was expressed (Supplementary Fig. 5d) indicating that in the absence of the nancompartment the imported iron was not sufficiently sequestered by the endogenous iron homeostasis machinery.
We furthermore tested variants of A with N-terminal fusions with peptide sequences from Magnetospirillum magneticum Mms (6 and 7) proteins reported to aid in templating iron mineralization $^{56}$ but found no additional benefit of these modified inner surfaces over A ${ }^{\text {FLAG }}$ using our current readout (Supplementary Fig. 5e). In addition, we analyzed several variants of the cargo proteins $\mathrm{B}$ and $\mathrm{C}$, fused $\mathrm{C}$-terminally to peptides from $\mathrm{Mms}$ proteins (superscripts M6, M7, please see Supplementary Fig. 5f). These data confirmed that either B or C are sufficient to load the nanocompartment with iron and showed that no obvious additional iron loading resulted from the presence of the Mms peptides.

Encapsulins enable detection via MRI and magnetic sorting. Next, we were interested in whether the strong iron accumulation inside eukaryotically expressed encapsulin shells would yield significant contrast by MRI. We thus expressed $A^{\text {FLAG }}$ alone or together with either all native cargos $\mathrm{BCD}_{\mathrm{P} 2 \mathrm{~A}}$ or just ${ }^{\mathrm{Myc}} \mathrm{B}$, or ${ }^{M{ }^{C}} \mathrm{D}$ and Zip14 and subjected cell pellets to relaxometry measurements by MRI. The nanocompartment $A^{\text {FLAG }}$ co-expressed with all native cargo proteins (BCD) lead to a significant increase in $R_{2}{ }^{*}$-relaxation rates as compared to just $A^{\text {FLAG. The same }}$ effect was observed by co-expressing just the ferritin-like $B$ (Fig. 7a, $p=0.0047$, Kruskal-Wallis with significant differences at $\alpha=0.05$ from Dunn's multiple comparisons test vs. $A^{\text {FLAG }}$, 
a
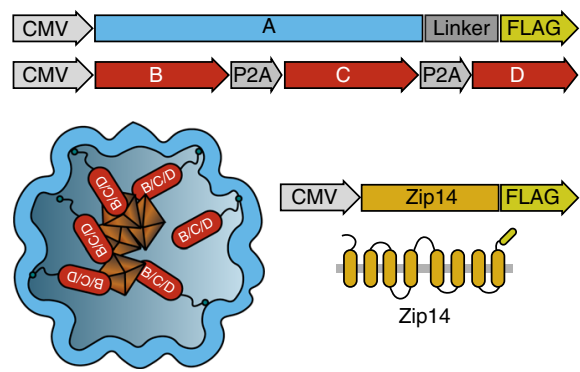

b

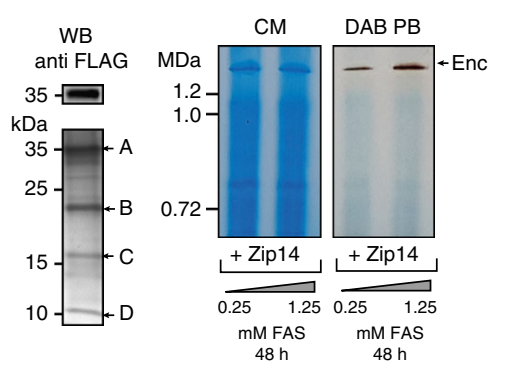

c
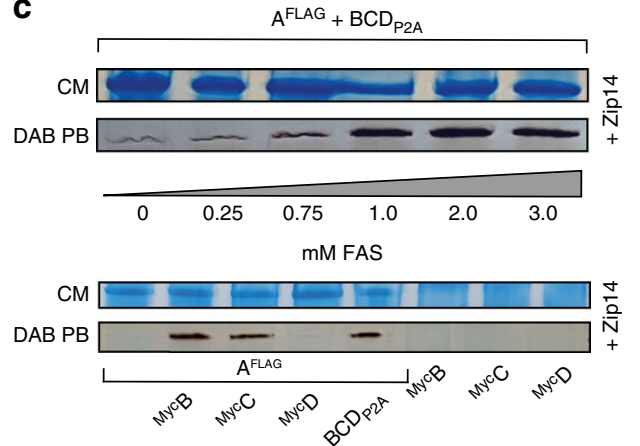

Fig. 6 Efficient iron loading of eukaryotically expressed encapsulin nanospheres a Schematic of a dual-promoter construct used for generation of a stable cell line expressing $A^{F L A G}$ and all native cargos B, C, and D. Also depicted is a construct encoding the iron-transporter MmZip14 ${ }^{\mathrm{FLAG}}$ (Zip14) used to transport additional amounts of iron into the cell. b Co-immunoprecipitation (Co-IP) against the FLAG epitope from a whole cell lysate of a stable HEK293T clone expressing $A^{F L A G}$ together with $B, C$, and D analyzed by silver-stained SDS PAGE and the corresponding WB against the FLAG epitope (left panel). The pair of Blue Native (BN) gels visualizes proteins from whole cell lysates via Coomassie staining (CM) (left panel) and iron content via treatment with DAB enhanced Prussian Blue (DAB PB) (right panel) from the same stable cell line. Robust iron loading of the assembled nanocompartments was achieved by transient co-expression of MmZip14 FLAG ${ }_{\text {IRES }}$-ZsGreen1 in which case $0.25 \mathrm{mM}$ ferrous ammonium sulfate (FAS) for $48 \mathrm{~h}$ was sufficient to see strong iron loading. c BN gel stained with CM or DAB PB loaded with whole cell lysates of HEK293T cells transiently expressing $A^{\text {FLAG }}+B C D$ P2A and Zip14 FLAG supplemented with different concentrations of FAS (0-3 mM) for $48 \mathrm{~h}$ (upper panel). The strong bands, which correspond to the assembled nanoshell, indicate high expression levels of encapsulins and efficient, dose-dependent iron loading. The lower panel shows a CM and DAB PB-stained BN gel from whole cell lysates of HEK293T cells expressing Zip14 ${ }^{\mathrm{FLAG}}$ and different combinations of native cargo molecules: ${ }^{\mathrm{Myc}} \mathrm{B}$, ${ }^{\mathrm{Myc} C}$, and ${ }^{\mathrm{Myc} D}$ alone, or all three $\left(B C D_{P 2 A}\right)$ with or without $A^{F L A G}$. The robust $D A B P B$ stains show that the ferritin-like cargo proteins $B$ or $C$ are sufficient for iron loading into encapsulins. FAS was supplemented at $2.5 \mathrm{mM}$ for $48 \mathrm{~h}$
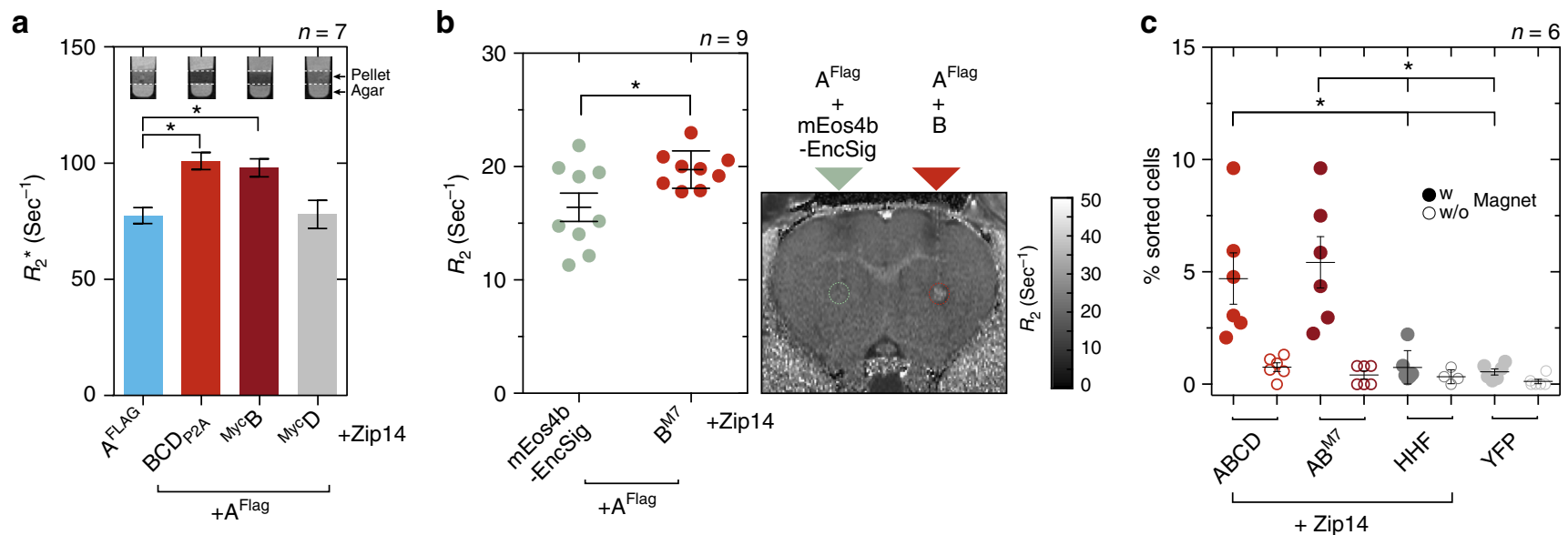

Fig. 7 Iron-filled encapsulins enable detection by MRI and magnetic cell separation. a Relaxometry measurements by MRI conducted on cell pellets $\left(\sim 10^{7}\right.$ cells) from HEK293T cells transiently expressing $A^{F L A G}+B C D_{P 2 A}, M^{M C B}$, or MyCD, or $A^{F L A G}$ alone (1 mM FAS for $24 \mathrm{~h}$ and expression of Zip14 ${ }^{\mathrm{FLAG}}$ ). Expression of $A^{F L A G}$ with $B C D_{P 2 A}$ or with ${ }^{M y C} B$ showed a significantly enhanced $R_{2}{ }^{*}$-relaxation rate as compared with $A^{F L A G}$ alone or loaded with Myc $D$; ferritin-like cargo $B$ was sufficient to generate an increase in $R_{2}{ }^{*}$ in the presence of the $A^{\text {FLAG }}$ nanocompartment $(p=0.0047$, Kruskal-Wallis, $n=7$ from four independent experiments, stars indicate significance at $\alpha=0.05$ from Dunn's multiple comparisons test vs. $A^{F L A G}$; the bars represent the mean \pm SEM). The insets show MRI slices ( $13.5 \mathrm{~ms}$ echo time) through test tubes in which cells were pelleted on a layer of agar. $\mathbf{b} \mathrm{In}$ vivo MRI detection of HEK293T cells transiently co-expressing $A^{F L A G}$ together with ferritin-like $B^{M 7}$ that were xenografted into rat brains. As compared to cells co-expressing $A^{\text {FLAG }}$ together with the fluorescent protein $\mathrm{mEos} 4 \mathrm{~b}$-EncSig as control cargo, we observed significantly increased transverse relaxation rates $(p=0.0078$, Wilcoxon matched-pairs signed rank test, $n=9$ ) measured at the injection site for $A^{\text {FLAG }}+B^{M 7}$ expressing cells $24 \mathrm{~h}$ post injection. The horizontal lines represent the mean $\pm S E M$. The image on the right shows a coronal $R_{2}$ map through a rat brain with the regions of interest (ROIs) defined over the injection sites by dashed circles. $\mathbf{c}$ HEK293T cells were co-expressing $A^{F L A G}$ and BCD ${ }_{P 2 A}, A^{F L A G}$ and $B^{M 7}$, or human $\mathrm{H}$-chain ferritin (HHF) as a control together with Zip14 and were treated with $2.5 \mathrm{mM}$ FAS for $48 \mathrm{~h}$. Additional control cells were expressing only EYFP. Independent cell suspensions were subsequently sorted on commercial magnetic separation columns inside and outside the magnetic field to control for unspecific retention in the mesh of the column. The fraction of cells separated in the magnetic field for both encapsulin:cargo conditions was significantly higher than for any of the control conditions HHF + Zip14 or YFP ( $p=0.0007$, Kruskal-Wallis, $n=6$ from three independent experiments, stars indicate significance at $\alpha=0.05$ from Dunn's multiple comparisons test across all conditions with magnetic field; the horizontal lines represent the mean \pm SEM) 
a

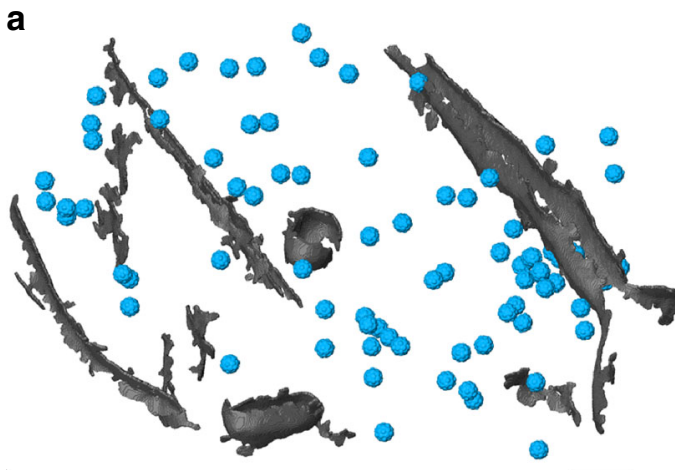

b

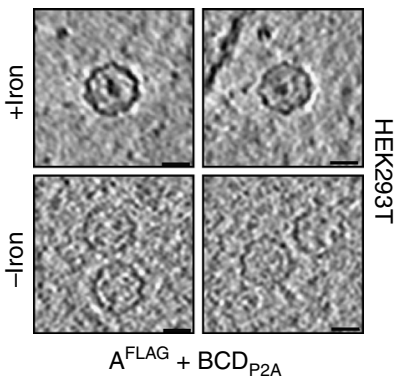

C

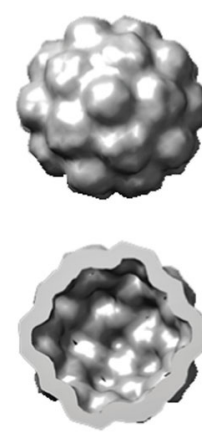

Structure from E. coli
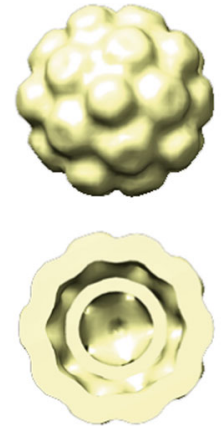

$A B C D$

untreated
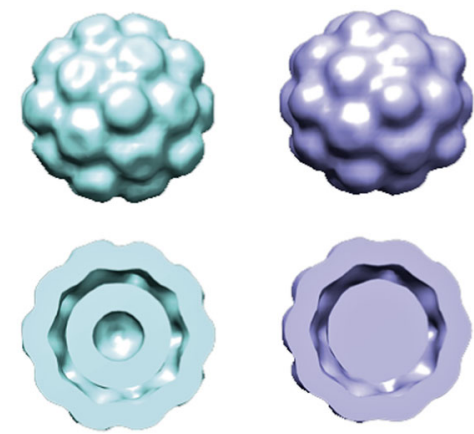

$A B C D$

0.25 mM FAS

+ transferrin

Iron loading

Fig. 8 Encapsulins as genetically encoded markers for cryo-electron tomography (Cryo-ET). a Cryo-ET data from HEK293T cells stably expressing encapsulins together with native ferritin-like cargo proteins (using the dual promoter construct $A^{F L A G ; B C D}{ }^{P 2 A}$ shown in Fig. 6a). 3D rendering showing encapsulins in blue and membranes in gray colors. Scale bar:100 nm. b Example slices from tomograms show encapsulins with and without electron-dense cores from iron-accumulation (treatment with $5 \mathrm{mM} \mathrm{FAS} \mathrm{for} 48 \mathrm{~h}$ prior to vitrification). Scale bars:20 nm. c In situ structures (displayed as $2 \times$ binned) derived from cryoelectron tomography of nanocompartments assembled in HEK293T cells without (beige), with $0.25 \mathrm{mM} \mathrm{FAS} \mathrm{and} 1 \mathrm{mg} / \mathrm{ml}$ human transferrin (cyan) and $5 \mathrm{mM} \mathrm{FAS} \mathrm{(purple)} \mathrm{as} \mathrm{compared} \mathrm{with} \mathrm{the} \mathrm{published} \mathrm{structure} \mathrm{shown} \mathrm{in} \mathrm{gray} \mathrm{(pdb} \mathrm{4PT2;} \mathrm{EMDataBank} \mathrm{EMD-5917)} \mathrm{that} \mathrm{was} \mathrm{obtained}$ from $M$. xanthus EncA expressed in $E$. coli ${ }^{31}$. The cutaway views of the encapsulins show electron densities indicating the presence of cargo proteins (beige) and additional iron deposition (cyan and purple) as compared to published data from the EncA shell ${ }^{31}$ that were obtained in the absence of cargo proteins

$n=7)$. This indicated again that co-expression of $\mathrm{B}$ was sufficient to generate efficient iron deposition inside the nanoshell.

We subsequently sought to test whether cells genetically labeled with encapsulins could be detected by MRI in vivo. As an initial assessment, we thus xenografted cells co-expressing $A^{\text {FLAG }}$ together with $B^{M 7}$ into rat brains and obtained $R_{2}$ relaxation maps that showed elevated relaxation rates $(p=0.0078$, Wilcoxon matched-pairs signed rank test, $n=9$ ) at the injection site as compared to xenografted cells in which the fluorescent protein mEos4b-EncSig was used as a control cargo (Fig. 7b).

In addition to MRI contrast, the iron biomineralization inside the encapsulins also allowed us to magnetically sort cells coexpressing the shell $A^{\text {FLAG }}$ with $\mathrm{BCD}_{\mathrm{P} 2 \mathrm{~A}}$ or with $\mathrm{B}^{\mathrm{M} 7}$ at significantly higher percentages than when human $\mathrm{H}$-chain ferritin (HHF) was expressed or just yellow fluorescent protein (EYFP) ( $p=0.0007$, Kruskal-Wallis, with significant differences at $\alpha=0.05$ from Dunn's multiple comparisons test, $n=6$; Fig. 7c).

Encapsulins as markers for electron microscopy. Given that the iron loading of the encapsulins was very efficient and observable at the population level, we next assessed how well individual nanocompartments could be detected by electron microscopy in cells such that they could be used as genetically encoded markers. We thus grew HEK293T cells stably expressing the shell protein
$\mathrm{A}^{\mathrm{FLAG}}$ and $\mathrm{BCD}_{\mathrm{P} 2 \mathrm{~A}}$ using a dual promoter vector on a transmission electron microscopy (TEM) grid, vitrified them by plunge-freezing and produced lamellae by cryo-focused ion beam (cryo-FIB) milling for in situ cellular cryo-electron tomography (cryo-ET). The heterologously expressed encapsulins were readily detected as clearly discernible nanospheres (Fig. 8a, Supplementary Fig. 6a, b) that exhibited electron-dense cores when we supplemented the growth media with ferrous iron (Fig. 8b) and were distributed as monodisperse spheres throughout the cytosol (Supplementary Fig. 6c, d). The electron density maps showed a high similarity to the structure published from encapsulin shells from M. xanthus expressed in E. coli (pdb 4PT2; EMDataBank EMD-591728, Fig. 8c). The clipped views from the encapsulins (blue) furthermore show electron densities associated with docked cargo proteins and most likely biomineralized iron as compared with the inner surface of the shell from E. coli (gray) that was mapped in the absence of any cargo (Fig. 8c, lower row). These data demonstrate that the spherical shape and high, nontoxic expression levels make encapsulin very attractive as fully genetically expressed markers for EM.

\section{Discussion}

In summary, we genetically controlled multifunctional orthogonal compartments in mammalian cells via expressing $\mathrm{N}-$ or 
C-terminally modified encapsulins, which we found to autoassemble into abundant nanocompartments which readily encapsulated sets of natural and engineered cargo proteins and enabled size-constrained metal biomineralization.

The efficiency of self-targeting and auto-packaging of the various cargo proteins in mammalian cells was remarkable given that the number of possible protein interactions is even a few-fold higher than in the original prokaryotic host organism ${ }^{57,58}$. We found that about 60 cargo proteins of a canonical fluorescent protein can be bound to the inner encapsulin surface via the minimal encapsulation signal. Higher loading factors could be achieved by providing cargo proteins with multidentate adapters such that the entire encapsulin volume could be filled. We furthermore observed that without co-expression of endogenous or engineered cargo, the abundance of the 60-mer encapsulin shell with $T=1$ symmetry was increased, which is in line with a previous observation made from $M$. xanthus encapsulin expressed in $E$. coli ${ }^{31}$ and suggests that encapsulation of cargo leads to the preferred assembly of the 180 -mer in $T=3$ symmetry.

Also, iron storage inside the capsule via the ferritin-like enzymes B or $\mathrm{C}$ targeted to the encapsulins was very efficient, indicating that there was sufficient access to ferrous iron. Whereas encapsulins heterologously expressed in E. coli were shown to load iron ${ }^{59}$, this could so far not been shown in mammalian cells. We also found that just co-expression of B (or C) with $\mathrm{A}$ is sufficient for robust iron storage such that a singlepiece reporter construct of just $\sim 2.1 \mathrm{~kb}$ in size can be used.

In the context of optimizing $T_{2}$ contrast in MRI, it would certainly be valuable to explore modifications of the outer surface that may control the agglomeration state and thus could modulate the apparent relaxivity of encapsulin ensembles ${ }^{60}$. In this context, it would also be desirable to explore capsid architectures with more storage capacity such as ones with $T=7$ quasisymmetry known from bacteriophage HK97 ${ }^{31,61}$. Furthermore, modifications of the inner surface of the shell may be engineered and/or additional cargo could be designed that could facilitate the nucleation process to support higher iron packing densities or alter environmental parameters (e.g., $\mathrm{pH}$ and redox potential) to potentially even generate superparamagnetic iron-oxides which possess a substantially larger magnetization ${ }^{62}$. Iterative optimization schemes such as directed evolution could also be employed based on rescue assays from excess iron or magnetic microfluidic sorting and could also be complemented by parallel screens in prokaryotes if enough iron-influx can be achieved there.

Their dense monodisperse distribution, spherical shape, and sufficient size, also render encapsulins excellent genetically expressed EM markers in mammalian cells (Supplementary Movie 1) that are much more readily detectable than ferritins, which have been visualized by EM in $E$. coli and yeast ${ }^{63,64}$. In addition, the iron-based contrast in encapsulins has the advantage over semi-genetic methods such as metallothionein (MT), miniSOG, erHRP, or APEX/APEX2 that no fixation and delivery of artificial substrates and precipitation of electron-dense material is necessary which may alter cellular structures ${ }^{53,65-68}$. Instead, if iron-based EM contrast is desired, cells expressing the ironaccumulating encapsulins can just be grown in regular growth media containing sufficient iron for transferrin-mediated uptake before direct plunge freezing and cryo-EM.

For future applications as EM gene reporters in, e.g., connectomics research, it would be desirable to generate further encapsulin variants with surface-presented targeting moieties to control their subcellular localization. In this regard, it is of note that virtue of the self-assembling mechanism, the size of A is only $0.9 \mathrm{~kb}$ and that of $\mathrm{B}$ just $0.5 \mathrm{~kb}$ such that a combined construct is small enough to be carried by viruses optimized for transsynaptic tracing ${ }^{69}$. It should furthermore be feasible to perform selective detection of encapsulins loaded with split photoactivatable fluorescent proteins via photoactivated localization microscopy (PALM) and combine this with cryo-ET as was demonstrated for photoactivatable GFP (cryo-PALM) ${ }^{70}$.

Besides allowing the influx of metals for size-constrained biomineralization for the type of applications discussed above, the pore size of $\sim 5 \AA$ inside the encapsulin shell also affords selective passage of small substrates, whereas reaction products may be trapped inside the nanoshell. We have exploited this feature by encapsulating tyrosinase for confined enzymatic production of the toxic polymer melanin and utilized the engineered "nanomelanosomes" as genetically encoded reporters for optoacoustic imaging.

In future applications, encapsulins could thus be used as versatile reaction chambers for, e.g., metabolic engineering of orthogonal reactions in eukaryotic cells. The toolbox for genetically controlled compartmentalization in mammalian cells which we introduce here could, for instance, enable multi-step enzymatic production involving labile or toxic intermediates but yielding end-products that may have beneficial intracellular effects or serve as molecular signals upon "quantal" release from the nanocompartment. The approach could for instance also endow genetically modified mammalian cells used for cell therapies with metabolic pathways that may augment their therapeutic efficacy. Complementarily, endogenously produced toxic products could be contained and detoxified in engineered compartments for causal studies or potentially for cell or gene therapies.

In addition to the encapsulins presented here, heterologous expression of compartments with different sizes and shapes seem possible, which could offer different sets of endogenous and engineered cargo molecules with different subcellular targeting. These alternative systems would ideally also be orthogonal to each other such that multiplexing (maybe even nesting) of several engineered compartments and multicomponent processes could be achieved.

More generally, genetically controlled compartmentalization of multi-component processes in eukaryotic cells-as demonstrated for encapsulins here-is a fundamental biotechnological capability that has profound implications for mammalian cell engineering and emerging cell therapies.

\section{Methods}

Genetic constructs. Mammalian codon-optimized MxEncA (UniProt: MXAN_3556) MxEncB, MxEncC, and MxEncD (UniProt: MXAN_3557, MXAN_4464, MXAN_2410) were custom synthesized by Integrated DNA Technologies and cloned into pcDNA $3.1(+)$ Zeocin (Invitrogen) using restriction cloning or Gibson assembly. The MxEncA surface tags (FLAG or StrepTagII) were C-terminally appended using Q $5^{\circledR}$ Site-Directed Mutagenesis (New England Biolabs). N-terminal Myc epitopes were added accordingly to the cargo proteins. Multigene expression of $\mathrm{B}, \mathrm{C}$, and $\mathrm{D}$ was achieved by generating a single reading frame containing all three genes separated by $\mathrm{P} 2 \mathrm{~A}$ peptides yielding $\mathrm{BCD}_{\mathrm{P} 2 \mathrm{~A}}$. A "scarless" bicistronic construct encoding ${ }^{\mathrm{My} C} \mathrm{C}$ and $\mathrm{A}^{\mathrm{STII}}$ was custom synthesized by inserting a $S s p$ DnaE mini-intein variant engineered for hyper-N-terminal autocleavage followed by a $\mathrm{P} 2 \mathrm{~A}$ peptide in between the genes as previously described $^{41}$. For generating stable clones expressing MxEncABCD, MxEncA ${ }^{\text {FLAG }}$ was cloned into the Cytomegalovirus promoter (CMV) driven expression cassette of pBudCE4.1 (Invitrogen) and $\mathrm{BCD}_{\mathrm{P} 2 \mathrm{~A}}$ was cloned into the elongation factor 1 alpha promoter (EF1a) driven expression cassette of the vector via restriction cloning. To generate AAV enabling multigene expression of MxEncA ${ }^{\text {FLAG }}$ and MxB-Mms7ct, two strategies were employed: MxEncA ${ }^{\text {FLAG }}$ was cloned upstream of an ECMV internal ribosome entry site (IRES) whereas MxB-Mms7ct was inserted downstream. The second approach employs MxB-Mms7ct followed by a

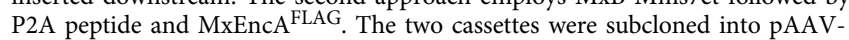
CamKIIa (https://www.addgene.org/26969/) with BamHI and EcoRI. AAVs were custom prepared by the UNC Vector Core of the University of North Carolina at Chapel Hill. To test the bicistronic expression constructs used for the AAVs in HEK293T cells, the cassettes were also sub-cloned into the pcDNA $3.1(+)$ Zeocin with EcoRI and NotI. To target PAmCherryl and mEos4b as cargo to the encapsulin nanocompartments, the fluorescent proteins were C-terminally fused to $2 \times$ GGGGS linkers followed by the minimal encapsulation signal LTVGSLRR 
(EncSig). To generate the destabilized version of mEos4b, the L106P mutant of FKBP12 (DD-N) ${ }^{43}$ was N-terminally appended to mEos4b-EncSig using GibsonAssembly yielding DD-mEos4b-EncSig. For complementation of split PAmCherryl inside the encapsulin nanoshell, amino acids 1-159 of PAmCherry1 were fused to MxEncC via a $2 \times$ GGGGS linker and amino acids $160-236$ of PAmCherry1 were directly fused to the $\mathrm{C}$-terminus of $\mathrm{MxEncB}$. For complementation of a split luciferase, the split part LgBit (NanoBiT system, Promega) was fused C-terminally to MxEncC via a $2 \times$ GGGGS linker. SmBit was directly fused to the C-terminus of MxEncB. SmCSE ${ }^{55}$ (UniProt: Smal_0489) and APEX2 ${ }^{53}$ were fused to $2 \times$ GGGGS linker followed by the minimal encapsulation signal. Mammalian codon-optimized Bacillus megaterium tyrosinase (BmTyr) was C-terminally appended to ${ }^{\mathrm{Myc}} \mathrm{D}$ separated by $2 \times$ GGGGS linker in custom gene synthesis. Cterminally FLAG-tagged Mus musculus Zip14 was inserted into pcDNA $3.1(+)$ or pIRES2-ZsGreenl via restriction cloning. To yield secreted encapsulins, MxEncA $^{\text {STII }}$ was $\mathrm{N}$-terminally fused to a human BM40 secretion peptide. In order to generate encapsulin derivatives featuring $\mathrm{C}$-terminal acidic peptides of magnetotactic bacteria Mms proteins that are implicated in mediation of magnetite formation either the Cterminal peptide of Mms6 (YAYMKSRDIESAQSDEEVELRDALA) or Mms7 (YVWARRRHGTPDLSDDALLAAAGEE) of Magnetospirillum magneticum were fused either to the inward-facing N-terminus of MxEncA ${ }^{\mathrm{FLAG}}$ or to the C-terminus of either the MxEncB or $\mathrm{C}$ using $\mathrm{Q}^{\oplus}{ }^{\oplus}$ Site-Directed Mutagenesis. For a complete list of the genetic constructs featuring their composition refer to Supplementary Table 1.

Cell culture. Low passage number HEK293T (ECACC: 12022001, obtained via Sigma-Aldrich) and CHO (ECACC: 85050302, obtained via Sigma-Aldrich) cells were cultured in advanced DMEM with $10 \%$ FBS and penicillin-streptomycin at $100 \mu \mathrm{g} / \mathrm{ml}$ at $37^{\circ} \mathrm{C}$ and $5 \% \mathrm{CO}_{2}$. Cells were transfected with X-tremeGENE HP (Roche) according to the protocol of the manufacturer. DNA amounts (ratio shell to cargos) were kept constant in all transient experiments to yield reproducible DNA-Lipoplex formation. To generate a stable HEK293T cell line expressing MxEncABCD, cells were transfected with pBudCE4.1 MxEncABCD and stable transfectants were selected with $300 \mu \mathrm{g} / \mathrm{ml}$ Zeocin (InvivoGen).

Protein expression and lysis. Cells were harvested between 24 and $48 \mathrm{~h}$ post transfection. Cells were lysed with M-PER Mammalian Protein Extraction Reagent (Pierce Biotechnology) containing a mammalian protease inhibitor cocktail (SIGMA P8340, Sigma-Aldrich) according to the protocol of the manufacturer in all experiments using FLAG-tagged encapsulins. For lysis of cells expressing StrepTagII-modified encapsulins, cells were resuspended in Buffer W $(150 \mathrm{mM}$ $\mathrm{NaCl}, 100 \mathrm{mM}$ Tris-Cl, $\mathrm{pH}$ 8.0) and exposed to four freeze-thaw cycles in $\mathrm{LN}_{2}$. After spinning down cell debris at $10,000 \times g$ for $15 \mathrm{~min}$, cell lysates were kept at $4{ }^{\circ}$ $\mathrm{C}$ for downstream analyses. Protein concentrations of lysates were determined by measuring $\mathrm{OD}$ at $280 \mathrm{~nm}$.

Co-immunoprecipitation of encapsulins. Cell lysates were incubated with AntiFLAG $^{\oplus}$ M2 Magnetic Beads or Anti-FLAG ${ }^{\oplus}$ M2 affinity gel (SIGMA M8823 and A2220, Sigma-Aldrich) according to the protocol of the manufacturer. After binding, the magnetic beads were washed four times on a magnetic separator rack (DYNAL separator, Invitrogen) with M-PER buffer. Bound FLAG-tagged encapsulins were eluted using M-PER buffer containing $100 \mu \mathrm{g} / \mathrm{ml}$ FLAG-peptide (SIGMA F3290, Sigma-Aldrich). In the case of encapsulins with an external StrepTagII, MagStrep "type3" XT beads or Strep-Tactin ${ }^{\circledR}$ XT resin (IBA Lifesciences) was used according to the protocol of the manufacturer. Proteins were eluted using Buffer BXT (150 mM NaCl, $100 \mathrm{mM}$ Tris-Cl, pH 8.0, $50 \mathrm{mM}$ Biotin). To analyze the eluted proteins, samples were mixed with SDS-PAGE sample buffer and incubated at $95^{\circ} \mathrm{C}$ for $5 \mathrm{~min}$. Samples were loaded onto pre-cast $12 \%$ Bio-Rad Mini-PROTEAN ${ }^{\circledR}$ TGX $^{\text {s" }}$ (Bio-Rad Laboratories) gels and run for $45 \mathrm{~min}$ at $200 \mathrm{~V}$. Accordingly, gels were either directly silver-stained using SilverQuest ${ }^{\text {six }}$ Silver Staining Kit (Novex) according to the protocol of the manufacturer or immunoblotted onto PVDF membranes. After blotting, membranes were blocked in 5\% non-fat milk in TBS for $1 \mathrm{~h}$ at room temperature. Subsequently, membranes were incubated in TBS containing 5\% non-fat milk and $1 \mu \mathrm{g} / \mathrm{ml}$ Monoclonal ANTIFLAG $^{\circledast}$ M2 antibody (SIGMA F1804, Sigma-Aldrich) or $1 \mu \mathrm{g} / \mathrm{ml}$ Anti-Myc Tag Antibody clone 9E10 (05-419, EMD Millipore) for $2 \mathrm{~h}$ at room temperature. After five washing cycles with TBS, membranes were incubated with anti-mouse IgG HRP-conjugate (SIGMA A5278, Sigma-Aldrich) for $1 \mathrm{~h}$ at room temperature in 5\% non-fat milk in TBS. Protein bands were detected using Amersham ECL Prime Western Blotting Detection Reagent (GE Healthcare Bio-Sciences AB) on a Fusion FX7/SL advance imaging system (Peqlab Biotechnologie GmbH). For dephosphorylation of protein material from the Co-IP, 10 units of calf intestinal phosphatase (New England Biolabs) were added to protein solutions in $1 \times$ CutSmart Buffer (New England Biolabs) and incubated for $1 \mathrm{~h}$ at $37^{\circ} \mathrm{C}$. For densitometric determination of SDS-PAGE bands, band intensity integrals were measured using Image (NIH)

Blue Native gel electrophoresis and on-gel analyses. For detection of native encapsulin nanocompartments, the NativePAGE ${ }^{m}$ Novex $^{\circledR}$ Bis-Tris Gel System (Life Technologies) was used. Either eluted material from the Co-IP/purification or whole cell lysates of cells expressing encapsulins in NativePAGE ${ }^{\mathrm{m}}$ Novex $^{\circledast}$ sample buffer were loaded onto pre-cast NativePAGE ${ }^{\mathrm{m}}$ Novex $^{\circledR} 3-12 \%$ Bis-Tris gels. NativeMark $^{\text {th }}$ Unstained Protein Standard (Life Technologies) covering a size range between 20 and $1200 \mathrm{kDa}$ was used as a marker. The total protein amount of whole cell lysates loaded per well was adjusted to $\sim 1-3 \mu$ g. Blue native (BN) gels were run for 90-180 min at $150 \mathrm{~V}$ according to the protocol of the manufacturer. Gels loaded with samples from Co-IP/purification were silver-stained using SilverQuest ${ }^{\mathrm{mm}}$ Silver Staining Kit (Novex) or Coomassie-stained using Bio-Safe ${ }^{m}$ Coomassie Stain (BioRad Laboratories). For protein detection, gels loaded with whole cell lysate samples were Coomassie-stained accordingly. For detection of iron-containing proteins, gels loaded with samples containing iron loaded encapsulins were Prussian Blue (PB) stained. Briefly, gels were incubated in $2 \%$ potassium hexacyanoferrate(II) in $10 \% \mathrm{HCl}$ for $45 \mathrm{~min}$. For 3,3'-diaminobenzidine-enhancement (DAB PB), gels were washed three times with $\mathrm{ddH}_{2} \mathrm{O}$ and incubated in $0.1 \mathrm{M}$ phosphate buffer $(\mathrm{pH}$ 7.4) containing $0.025 \% \mathrm{DAB}$ and $0.005 \% \mathrm{H}_{2} \mathrm{O}_{2}$ until dark-brown bands appeared. To stop DAB polymerization, gels were washed three times with $\mathrm{ddH}_{2} \mathrm{O}$. For detection of fluorescent signals from native encapsulin bands (fluorescent cargos: mEos4b, PAmCherryl, split PAmCherry1 or mineralized CdS), unstained BN gels were imaged on a Fusion FX7/SL advance imaging system (Peqlab Biotechnologie $\mathrm{GmbH}$ ) using the UV fluorescence mode. For on-gel detection of luminescence signal generated by encapsulated split NanoLuciferase, unstained BN gels were soaked in $1 \mathrm{ml}$ of Nano-Glo ${ }^{\oplus}$ Luciferase substrate (Nano-Glo ${ }^{\circledast}$ Luciferase Assay, Promega) and imaged on a Fusion FX7/SL advance imaging system (Peqlab Biotechnologie $\mathrm{GmbH}$ ) in chemiluminescence mode. For whole cell lysate luminescence detection, cell lysates were mixed with the substrate at a 1:1 ratio and luminescence readings were taken on a Centro LB 960 (Berthold Technologies) at $0.1 \mathrm{~s}$ acquisition time. For detection of APEX2 peroxidase activity inside encapsulins, unstained $\mathrm{BN}$ gels were incubated in $0.1 \mathrm{M}$ phosphate buffer ( $\mathrm{pH} 7.4)$ containing $0.025 \% \mathrm{DAB}$ and $0.005 \% \mathrm{H}_{2} \mathrm{O}_{2}$ for 15 min until black bands appeared on the gel. For microscopic detection of DAB polymerization in cells expressing APEX2-loaded encapsulins, cells were fixed in 4\% PFA in PBS for $15 \mathrm{~min}$. Subsequently, cells were incubated in $0.1 \mathrm{M}$ phosphate buffer $(\mathrm{pH}$ 7.4) containing $0.025 \% \mathrm{DAB}$ and $0.005 \% \mathrm{H}_{2} \mathrm{O}_{2}$ for $5 \mathrm{~min}$. The reaction was stopped by washing three times with PBS. For the on-gel detection of melanin generation associated with encapsulins, gels loaded with whole cell lysates of HEK293T cells expressing encapsulins loaded with tyrosinase were incubated in PBS containing $2 \mathrm{mM} \mathrm{L-}$ tyrosinase and $100 \mu \mathrm{M} \mathrm{CuCl}_{2}$ for $1 \mathrm{~h}$ at $37^{\circ} \mathrm{C}$ until a black encapsulin band became visible.

Size exclusion chromatography. Size exclusion chromatography (SEC) of purified $\mathrm{A}^{\mathrm{FLAG}}$ with or without DD-mEos4b-EncSig was performed on an Äkta Purifier (GE Healthcare) equipped with an analytical size exclusion column (Superose $610 /$ $300 \mathrm{GL}, \mathrm{GE}$ Healthcare) at $4{ }^{\circ} \mathrm{C}$. For refractive index (RI) detection, a Viscotek TDA 305 triple array detector (Malvern Instruments) downstream of the column was used. In total, $100 \mu \mathrm{l}$ samples were run at a flow rate of $0.4 \mathrm{ml} / \mathrm{min}$ in $50 \mathrm{mM}$ Tris- $\mathrm{HCl}, 150 \mathrm{mM} \mathrm{NaCl}, 1 \mathrm{mM}$ EDTA, $\mathrm{pH} 7.4$, at a concentration of $0.3 \mathrm{mg} / \mathrm{ml}$.

Dynamic light scattering. Dynamic light scattering experiments were performed on a DynaPro NanoStar instrument and analyzed with DYNAMICS 7.1.9 software (Wyatt Technology). Measurements were performed at $22^{\circ} \mathrm{C}$ using standard rectangular cuvettes containing $60 \mu \mathrm{l}$ of protein sample in the concentration range between 0.15 and $0.5 \mathrm{mg} / \mathrm{ml}$. For each measurement, 100 acquisitions with an acquisition time of $5 \mathrm{~s}$ were recorded.

Native mass spectrometry. Purified sample material from HEK293T cells expressing $A^{\mathrm{FLAG}}$, with and without co-expression of the photoactivatable fluorescent protein DD-mEos4b-EncSig, was buffer exchanged to $150 \mathrm{mM}$ aqueous ammonium acetate, pH 7.5 using Micro Bio-Spin Columns with Bio-Gel P6 (Biorad, USA) following the manufacturer's protocol for buffer exchange. Samples were analyzed at a concentration of $0.1-0.45 \mathrm{~g} / \mathrm{l}$, corresponding to an estimated monomer concentration ranging from 3 to $14 \mu \mathrm{M}$. Gold-coated nanoelectrospray needles were made in-house from borosilicate capillaries (Kwik-Fil, World Precision Instruments, Sarasota, FL) on a P97 puller (Sutter Instruments, Novato, CA) and being coated by using an Edwards Scancoat six pirani 501 sputter coater (Edwards Laboratories, Milpitas, USA). Measurements were carried out in positive ion mode on a modified Q-ToF 2 (Waters, UK) instrument ${ }^{71,72}$, operated at elevated pressure in the source region ( 10 mbar), using Xenon as collision gas at $2^{\star} 10^{-2}$ mbar in the collision cell. Capillary and sample cone voltage was set to $1400 \mathrm{~V}$ and $150 \mathrm{~V}$, respectively. The voltage before the collision cell was either set to $100 \mathrm{~V}$ or $250-300 \mathrm{~V}$, optimizing for desolvation of the intact complex or the subsequent ejection of subunits, respectively. Spectra were calibrated using an aqueous solution of cesium iodide $(25 \mathrm{mg} / \mathrm{ml})$ and exported from MassLynx. All further data analysis was performed with in-house developed python scripts (Python 3.6). When applicable, charges were assigned to charge state resolved peak series by extracting the top position for consecutive charge states and minimizing the standard deviation (SD) of the average mass by trying different charge states. Centroids for empty and cargo filled encapsulins $(T=3)$ were calculated using all data points above $40 \%$ of the base peaks intensity in the appropriate region $(\mathrm{m} / z$ $30,000-40,000$ for empty and $m / z 35,000-45,000$ for cargo filled encapsulins). The average was taken over three technical replicates and the error represents the 
standard deviation (SD). To estimate the mass from the $\mathrm{m} / \mathrm{z}$ position, we fitted 77 empirical determined masses and their corresponding $\mathrm{m} / z$ positions to the equation Mass $[\mathrm{kDa}]=A^{*} \mathrm{~m} / \mathrm{z}^{B}$. These 77 proteins consist of encapsulin $T=1$, which mass was determined in this study as well as 76 other assemblies, which were already measured and reported in previous publications $\mathrm{s}^{73,74}$. The resulting formula Mass $[\mathrm{kDa}]=1.63^{*} 10^{-6 *} \mathrm{~m} / \mathrm{z}^{2.14}$ was used to calculate the mass from the average $\mathrm{m} / \mathrm{z}$ positions of empty and cargo filled encapsulins $(T=3)$. Since mass and $\mathrm{m} / \mathrm{z}$ positions do not follow a linear relation, we averaged the upper and lower error from the $m / z$ dimension projected in the mass domain. Cargo load was estimated based on the difference between the predicted masses of empty and cargo loaded encapsulins $(T=3)$. The error of the mass difference was calculated using the equation $\sigma \Delta$ Mass $=\left(\sigma \text { Mass }^{2}+\sigma \text { Mass }^{2}\right)^{1 / 2}$. The difference in mass was then divided by $41.4 \mathrm{kDa}$, the mass of the DD-mEos $4 \mathrm{~b}$-EncSig monomer protein. The $\mathrm{A}^{\mathrm{FLAG}}$ monomer mass was calculated as the weighted average for the different proteoforms, using the summed intensities over the charge states for each species.

Complementation of split PAmCherry1 inside encapsulins. Cells transfected with C-PAs1 and B-PAs2 with or without $\mathrm{A}^{\mathrm{FLAG}}$ were seeded onto 8-well Poly-Llysine-coated microscopy chips (Ibidi). Thirty-six hours post transfection, live cell confocal microscopy was conducted on a Leica SP5 system (Leica Microsystems). For photoactivation of split PAmCherryl, samples were illuminated with a $405 \mathrm{~nm}$ laser for $60 \mathrm{~s}$ at $40 \%$ laser power. The signal of complemented split PAmCherry1 was excited using the $561 \mathrm{~nm}$ laser. To quantify the complementation of split PAmCherry 1 with or without the encapsulin shell, the ratio of the total mean fluorescence after photoactivation divided by the signal before was calculated. ImageJ was used to quantify mean fluorescence values from randomly chosen areas on the well.

Multispectral optoacoustic tomography. Optoacoustic images of cells coexpressing $\mathrm{A}^{\mathrm{STII}}$ and ${ }^{\mathrm{Myc}} \mathrm{D}$-BmTyr were acquired on an inVision 256-TF system (iThera Medical GmbH). Briefly, $\sim 10^{7}$ HEK293T cells co-expressing the genes treated with $10 \mu \mathrm{M} \mathrm{CuCl}_{2}$ and $1 \mathrm{mM} \mathrm{L}$-tyrosine $24 \mathrm{~h}$ prior to the measurement were detached using trypsin, washed with PBS, embedded into $1 \%$ low melting agar yielding a tubular phantom of $\sim 300 \mu \mathrm{l}$ volume. The cell phantom and an ink phantom $(\mathrm{OD}=0.2)$ were placed in a custom-built sample holder and optoacoustic images were acquired for the range of wavelengths between 690 and 900 $\mathrm{nm}$. Signals were reconstructed using ViewMSOT software suite (iThera Medical $\mathrm{GmbH}$ ) and linearly unmixed using a reference spectrum for melanin

Magnetic sorting. Cells were washed twice with PBS, detached with Accutase ${ }^{\circledast}$ (Sigma-Aldrich) and resuspended in DPBS supplemented with $10 \%$ fetal bovine serum (Gibco) prior to sorting. For magnetic sorting, columns filled with ferromagnetic spheres (MS columns, Miltenyi Biotec) were placed in an external magnetic field (OctoMACS separator, Miltenyi Biotec) and equilibrated with $1 \mathrm{ml}$ DPBS containing $10 \%$ FBS. The column was loaded with cells and washed with 0.5 ml DPBS; the flow-through was collected as one fraction. After removing the column from the magnetic separator, cells were eluted with $1 \mathrm{ml}$ DPBS. The total number of cells before sorting as well as the cell numbers in flow-through and eluate were determined with a Countess II FL Automated Cell Counter (Life Technologies).

Magnetic resonance imaging of cells. MR images were acquired at a Bruker BioSpec 94/20USR, 9.4T system equipped with a RF RES $4001 \mathrm{H}$ 112/072 Quad TR $\mathrm{AD}$ resonator. For $T_{2}^{*}$ measurements of cell pellets, $4^{*} 10^{6}$ HEK293T cells were seeded $24 \mathrm{~h}$ prior to transfection on poly-L-lysine-coated $10 \mathrm{~cm}$ cell culture dishes. Twenty four hours post transfection, ferrous ammonium sulfate (FAS) was added to the medium yielding a concentration of $1 \mathrm{mM}$. Twenty four hours post iron addition, cells were washed three times with DPBS and detached with Accutase ${ }^{\circledast}$ and centrifuged at $500 \times g$ for $4 \mathrm{~min}$. The pellets were resuspended in $800 \mu \mathrm{LPBS}$ and transferred to cryobank vials (Thermo Scientific Nunc) containing $50 \mu \mathrm{l}$ of solidified $1 \%$ agarose at the bottom. Cells were then spun down at $2000 \times g$ for 2 min and immediately used for MRI. $T_{2}{ }^{*}$ measurements were conducted in a custom-made holder filled with DPBS to avoid susceptibility artifacts. $T_{2}^{*}$ values were calculated based on a multiple gradient echo (MGE) sequence with a TR of $800 \mathrm{~ms}, 12$ echoes with an echo spacing of $4.5 \mathrm{~ms}(3.5-58.5 \mathrm{~ms})$, a flip angle of $50^{\circ}$, field of view of $65 \times 65 \mathrm{~mm}$ and a matrix size of $256 \times 256$. Relaxation rates were calculated with the Image Sequence Analysis Tool from Bruker BioSpin MRI $\mathrm{GmbH}$.

In vivo expression of encapsulins in murine brains. Mice were positioned in a stereotaxic frame, anesthetized with isoflurane, and implanted bilaterally with MRI compatible guide cannulae (Plastics One) that were stably fixated with dental cement. Injection cannulae (Plastics One) were connected via polyethylene tubing (PE-50), filled with silicone oil, connected to a PhD 2000 syringe pump (Harvard Apparatus) and backfilled with solutions containing AAV viral particles. Injection cannulae were inserted into the guide cannulae and lowered into the brain. A volume of $1 \mu \mathrm{l}$ of viral particles was injected at $0.1 \mu \mathrm{l} / \mathrm{min}$. Injection cannulae were subsequently retracted slowly and replaced with dummy cannulae (Plastics One) that screwed firmly into the guide cannula pedestals. All experiments on mice were conducted in accordance with the guidelines approved by the government of Upper Bavaria.

Immunohistochemistry. Three to six weeks after intracranial viral injection, mice were terminally anesthetized, perfused, and the brains were removed for cryosectioning. Brain slices were then blocked in SuperBlock (TBS) Blocking Buffer (Thermo Fisher Scientific) for $1 \mathrm{~h}$ at room temperature in a humidified chamber Subsequently, brain slices were incubated in $5 \mu \mathrm{g} / \mathrm{ml}$ Monoclonal ANTI-FLAG ${ }^{\circledR}$ M2 antibody (SIGMA F1804, Sigma-Aldrich) in TBS for $2 \mathrm{~h}$ at room temperature. After $5 \times 5 \mathrm{~min}$ washes with TBS, the brain slices were incubated in $1 \mu \mathrm{g} / \mathrm{ml}$ Goat anti-Mouse IgG $(\mathrm{H}+\mathrm{L})$ Cross-Adsorbed Secondary Antibody conjugated to Alexa Fluor 488 (A-11001, Invitrogen) in TBS for $1 \mathrm{~h}$ at room temperature in darkness. For nuclear counterstaining, DAPI was added at $300 \mathrm{nM}$ for 5 min. Finally, the brains slices were washed five times with TBS. Brain slices were subsequently imaged on an EVOS FL Auto Cell Imaging System (Invitrogen) or a Leica SP 5 confocal microscope (Leica Microsystems).

In vivo MRI. HEK293T cells $\left(\sim 4^{\star} 10^{6}\right)$ were seeded onto poly-L-lysine-coated $10 \mathrm{~cm}$ cell culture dishes. Twenty four hours after seeding, cells were transiently transfected at $70-80 \%$ confluency with DNA constructs encoding either $A^{\mathrm{FLAG}}+\mathrm{B}^{\mathrm{M} 7}$ or $\mathrm{A}^{\mathrm{FLAG}}+$ mEos4b-EncSig, as well as for both conditions Zip14 at $5 \%$ of the total DNA amount using X-tremeGENE ${ }^{\mathrm{mm}}$ (Roche). Twenty four hours post transfection, the cell culture medium was replenished with fresh medium containing 1 mM FAS. Twenty four hours after incubation with FAS, cells were washed gently three times with PBS, detached from the culture dishes after 5 min of treatment with a 1:1 solution of Accutase ${ }^{\circledast}$ (Sigma) and Trypsin, centrifuged for 5 min at $1200 \times g$ and resuspended in growth media. Cell suspensions were backfilled into two injection cannulae (28 Gauge, Plastics One, Roanoke, VA, USA) connected via plastic tubing to $25 \mu \mathrm{l}$ Hamilton glass syringes clamped in a remote dual syringe pump (PHD 22/2,000; Harvard Apparatus, Holliston, MA, USA). Injection cannulae (the side of injection for $\mathrm{A}^{\mathrm{FLAG}}+\mathrm{B}^{\mathrm{M} 7}$ or control were switched between experiments) were then lowered into bilateral guide cannulae (22 Gauge, Plastics One, Roanoke, VA) that were previously implanted in Sprague-Dawley rats ${ }^{75}$. Rats were then centered in the bore of a 7T $20 \mathrm{~cm}$ inner diameter, horizontal bore magnet (Bruker BioSpin MRI GmbH, Ettlingen, Germany) and gradient echo scans $(\mathrm{FOV}=2.5 \mathrm{~cm} \times 2.5 \mathrm{~cm}$, matrix size $=256 \times 256$; seven slices with $1 \mathrm{~mm}$ slice thickness) were taken at a TR $=800 \mathrm{~ms}$ and different echo times $(5,10,15,20,25$ $\mathrm{ms}$ ) to compute relaxation rate maps and perform ROI analysis (circular ROIs of 1 $\mathrm{mm}$ diameter placed on injection sites) using custom routines in Matlab (Mathworks, Natick, MA, USA). All procedures on rats were conducted in accordance with National Institutes of Health guidelines and with the approval of the MIT Committee on Animal Care.

Cell viability assays. Iron-related cytotoxicity was monitored via the Roche Cytotoxicity Detection Kit (LDH) (Roche Diagnostics) according to the protocol of the manufacturer. Briefly, $7.5^{*} 10^{5}$ HEK293T cells were seeded on poly-L-lysinecoated 24-well plates. Twenty four hours post seeding, cells were transfected with different combinations of genes using X-tremeGENE HP (Roche). The Zip14 DNA amount was kept constant in all samples expressing Zip14 (5\% of total DNA). For expression of combinations of $A^{\mathrm{FLAG}}$ with cargo proteins, $60 \%$ of the total DNA amount was encoding $\mathrm{A}^{\mathrm{FLAG}}$ and the remaining $35 \%$ were used for the respective cargo molecule. $24 \mathrm{~h}$ post transfection, FAS was added to the medium from a $100 \mathrm{mM}$ stock solution yielding a final concentration of $2.5 \mathrm{mM}$. Twenty four hours post addition of FAS, cells were assayed for LDH release. In order to evaluate gene-related toxicity in the absence of iron, the assay was performed accordingly but without iron supplementation and cells were assayed $48 \mathrm{~h}$ post transfection. The Luciferase-based viability assay (RealTime-Glo ${ }^{\mathrm{mm}}$ MT Cell Viability Assay, Promega) was performed according to the protocol of the manufacturer in 96-well plate format as an endpoint measurement. Luminescence readings were taken on a Centro LB 960 (Berthold Technologies) at $0.5 \mathrm{~s}$ acquisition time.

Electron microscopy. Please refer to Supplementary Methods in the Supplementary Information for a detailed description of the electron microscopy techniques used.

Data availability. Data are available upon reasonable request to the corresponding author. The cryo-EM maps of non-iron loaded and iron loaded encapsulins in HEK293T cells have been deposited under EMDB-4392 and 4393 respectively.

Received: 4 December 2017 Accepted: 16 April 2018

Published online: 18 May 2018

\section{References}

1. DeLoache, W. C. \& Dueber, J. E. Compartmentalizing metabolic pathways in organelles. Nat. Biotechnol. 31, 320-321 (2013). 
2. Küchler, A., Yoshimoto, M., Luginbühl, S., Mavelli, F. \& Walde, P. Enzymatic reactions in confined environments. Nat. Nanotechnol. 11, 409-420 (2016).

3. Murat, D., Quinlan, A., Vali, H. \& Komeili, A. Comprehensive genetic dissection of the magnetosome gene island reveals the step-wise assembly of a prokaryotic organelle. Proc. Natl Acad. Sci. USA 107, 5593-5598 (2010).

4. Lohße, A. et al. Functional analysis of the Magnetosome Island in Magnetospirillum gryphiswaldense: the mamAB operon is sufficient for magnetite biomineralization. PLoS ONE 6, e25561 (2011).

5. Heinhorst, S. \& Cannon, G. C. A new, leaner and meaner bacterial organelle. Nat. Struct. Mol. Biol. 15, 897-898 (2008).

6. Huber, M. C. et al. Designer amphiphilic proteins as building blocks for the intracellular formation of organelle-like compartments. Nat. Mater. 14, 125-132 (2015).

7. Avalos, J. L., Fink, G. R. \& Stephanopoulos, G. Compartmentalization of metabolic pathways in yeast mitochondria improves the production of branched-chain alcohols. Nat. Biotechnol. 31, 335-341 (2013).

8. O’Neil, A., Prevelige, P. E., Basu, G. \& Douglas, T. Coconfinement of fluorescent proteins: spatially enforced communication of GFP and mCherry encapsulated within the P22 capsid. Biomacromolecules 13, 3902-3907 (2012).

9. Zschoche, R. \& Hilvert, D. Diffusion-limited cargo loading of an engineered protein container. J. Am. Chem. Soc. 137, 16121-16132 (2015).

10. Seebeck, F. P., Woycechowsky, K. J., Zhuang, W., Rabe, J. P. \& Hilvert, D. A simple tagging system for protein encapsulation. J. Am. Chem. Soc. 128, 4516-4517 (2006).

11. Rurup, W. F., Snijder, J., Koay, M. S. T., Heck, A. J. R. \& Cornelissen, J. J. L. M. Self-sorting of foreign proteins in a bacterial nanocompartment. J. Am. Chem. Soc. 136, 3828-3832 (2014).

12. Minten, I. J., Hendriks, L. J. A., Nolte, R. J. M. \& Cornelissen, J. J. L. M. Controlled encapsulation of multiple proteins in virus capsids. J. Am. Chem. Soc. 131, 17771-17773 (2009).

13. Comellas-Aragonès, $M$. et al. A virus-based single-enzyme nanoreactor. Nat. Nanotechnol. 2, 635-639 (2007).

14. Azuma, Y., Zschoche, R., Tinzl, M. \& Hilvert, D. Quantitative packaging of active enzymes into a protein cage. Angew. Chem. Int. Ed. Engl. 55, 1531-1534 (2016).

15. Frey, R., Hayashi, T. \& Hilvert, D. Enzyme-mediated polymerization inside engineered protein cages. Chem. Commun. 52, 10423-10426 (2016).

16. Patterson, D. P., Prevelige, P. E. \& Douglas, T. Nanoreactors by programmed enzyme encapsulation inside the capsid of the bacteriophage P22. ACS Nano 6, 5000-5009 (2012).

17. Patterson, D. P., Schwarz, B., Waters, R. S., Gedeon, T. \& Douglas, T. Encapsulation of an enzyme cascade within the bacteriophage P22 virus-like particle. ACS Chem. Biol. 9, 359-365 (2014).

18. Giessen, T. W. \& Silver, P. A. A catalytic nanoreactor based on in vivo encapsulation of multiple enzymes in an engineered protein nanocompartment. ChemBioChem 17, 1931-1935 (2016).

19. Choudhary, S., Quin, M. B., Sanders, M. A., Johnson, E. T. \& Schmidt-Dannert, C. Engineered protein nano-compartments for targeted enzyme localization. PLoS ONE 7, e33342 (2012).

20. Held, M. et al. Engineering formation of multiple recombinant Eut protein nanocompartments in E. coli. Sci. Rep. 6, 24359 (2016)

21. Cai, F., Sutter, M., Bernstein, S. L., Kinney, J. N. \& Kerfeld, C. A. Engineering bacterial microcompartment shells: chimeric shell proteins and chimeric carboxysome shells. ACS Synth. Biol. 4, 444-453 (2015).

22. Kedersha, N. L., Heuser, J. E., Chugani, D. C. \& Rome, L. H. Vaults. III. Vault ribonucleoprotein particles open into flower-likestructures with octagonal symmetry. J. Cell Biol. 112, 225-235 (1991).

23. Kickhoefer, V. A. et al. Engineering of vault nanocapsules with enzymatic and fluorescent properties. Proc. Natl Acad. Sci. USA 102, 4348-4352 (2005).

24. Slesina, M. et al. Movement of vault particles visualized by GFP-tagged major vault protein. Cell Tissue Res. 324, 403-410 (2006).

25. Wang, M., Abad, D., Kickhoefer, V. A., Rome, L. H. \& Mahendra, S. Vault nanoparticles packaged with enzymes as an efficient pollutant biodegradation technology. ACS Nano 9, 10931-10940 (2015).

26. Tanaka, H. \& Tsukihara, T. Structural studies of large nucleoprotein particles, vaults. Proc. Jpn Acad. Ser. B Phys. Biol. Sci. 88, 416-433 (2012).

27. Gálvez, N. et al. Comparative structural and chemical studies of ferritin cores with gradual removal of their iron contents. J. Am. Chem. Soc. 130, 8062-8068 (2008).

28. Papaefthymiou, G. C. The Mössbauer and magnetic properties of ferritin cores. Biochim. Biophys. Acta 1800, 886-897 (2010).

29. Gossuin, Y., Gillis, P., Hocq, A., Vuong, Q. L. \& Roch, A. Magnetic resonance relaxation properties of superparamagnetic particles. Wiley Interdiscip. Rev. Nanomed. Nanobiotechnol. 1, 299-310 (2009).

30. Douglas, T. et al. Protein engineering of a viral cage for constrained nanomaterials synthesis. Adv. Mater. 14, 415 (2002).
31. McHugh, C. A. et al. A virus capsid-like nanocompartment that stores iron and protects bacteria from oxidative stress. EMBO J. 33, 1896-1911 (2014).

32. Cassidy-Amstutz, C. et al. Identification of a minimal peptide tag for in vivo and in vitro loading of encapsulin. Biochemistry 55, 3461-3468 (2016).

33. He, D. et al. Structural characterization of encapsulated ferritin provides insight into iron storage in bacterial nanocompartments. Elife 5, e18972 (2016).

34. Sutter, M. et al. Structural basis of enzyme encapsulation into a bacterial nanocompartment. Nat. Struct. Mol. Biol. 15, 939-947 (2008).

35. Moon, H., Lee, J., Min, J. \& Kang, S. Developing genetically engineered encapsulin protein cage nanoparticles as a targeted delivery nanoplatform. Biomacromolecules 15, 3794-3801 (2014).

36. Moon, $\mathrm{H}$. et al. Genetically engineering encapsulin protein cage nanoparticle as a SCC-7 cell targeting optical nanoprobe. Biomater. Res. 18, 21 (2014).

37. Putri, R. M., Fredy, J. W., Cornelissen, J. J. L. M., Koay, M. S. T. \& Katsonis, N. Labelling bacterial nanocages with photo-switchable fluorophores. ChemPhysChem 17, 1815-1818 (2016).

38. Kanekiyo, M. et al. Rational design of an Epstein-Barr virus vaccine targeting the receptor-binding site. Cell 162, 1090-1100 (2015).

39. Giessen, T. W. \& Silver, P. A. Converting a natural protein compartment into a nanofactory for the size-constrained synthesis of antimicrobial silver nanoparticles. ACS Synth. Biol. 5, 1497-1504 (2016).

40. Tamura, A. et al. Packaging guest proteins into the encapsulin nanocompartment from Rhodococcus erythropolis N771. Biotechnol. Bioeng. 112, 13-20 (2015)

41. Zhang, B. et al. Coordinated protein co-expression in plants by harnessing the synergy between an intein and a viral 2A peptide. Plant Biotechnol. J. 15, 718-728 (2017)

42. Paez-Segala, M. G. et al. Fixation-resistant photoactivatable fluorescent proteins for CLEM. Nat. Methods 12, 215-218 (2015). 4 p following 218.

43. Maynard-Smith, L. A., Chen, L.-C., Banaszynski, L. A., Ooi, A. G. L. \& Wandless, T. J. A directed approach for engineering conditional protein stability using biologically silent small molecules. J. Biol. Chem. 282, 24866-24872 (2007)

44. Nickerson, A., Huang, T., Lin, L.-J. \& Nan, X. Photoactivated localization microscopy with bimolecular fluorescence complementation (BiFC-PALM) for nanoscale imaging of protein-protein interactions in cells. PLOS ONE 9, e100589 (2014)

45. Dixon, A. S. et al. NanoLuc complementation reporter optimized for accurate measurement of protein interactions in cells. ACS Chem. Biol. 11, 400-408 (2016).

46. Stritzker, J. et al. Vaccinia virus-mediated melanin production allows MR and optoacoustic deep tissue imaging and laser-induced thermotherapy of cancer. Proc. Natl Acad. Sci. USA 110, 3316-3320 (2013).

47. Jathoul, A. P. et al. Deep in vivo photoacoustic imaging of mammalian tissues using a tyrosinase-based genetic reporter. Nat. Photonics 9, 239-246 (2015).

48. Ntziachristos, V. Going deeper than microscopy: the optical imaging frontie in biology. Nat. Methods 7, 603-614 (2010).

49. Ntziachristos, V. \& Razansky, D. Molecular imaging by means of multispectral optoacoustic tomography (MSOT). Chem. Rev. 110, 2783-2794 (2010).

50. Raposo, G. \& Marks, M. S. Melanosomes--dark organelles enlighten endosomal membrane transport. Nat. Rev. Mol. Cell Biol. 8, 786-797 (2007)

51. Hasegawa, T. Tyrosinase-expressing neuronal cell line as in vitro model of Parkinson's disease. Int. J. Mol. Sci. 11, 1082-1089 (2010).

52. Sendovski, M., Kanteev, M., Ben-Yosef, V. S., Adir, N. \& Fishman, A. First structures of an active bacterial tyrosinase reveal copper plasticity. J. Mol. Biol. 405, 227-237 (2011).

53. Lam, S. S. et al. Directed evolution of APEX2 for electron microscopy and proximity labeling. Nat. Methods 12, 51-54 (2015).

54. Wittig, I., Braun, H.-P. \& Schägger, H. Blue native PAGE. Nat. Protoc. 1, 418-428 (2006).

55. Dunleavy, R., Lu, L., Kiely, C. J., McIntosh, S. \& Berger, B. W. Single-enzyme biomineralization of cadmium sulfide nanocrystals with controlled optical properties. Proc. Natl Acad. Sci. USA 113, 5275-5280 (2016).

56. Jones, S. R. et al. Genetic and biochemical investigations of the role of MamP in redox control of iron biomineralization in Magnetospirillum magneticum. Proc. Natl Acad. Sci. USA 112, 3904-3909 (2015).

57. Kerner, M. J. et al. Proteome-wide analysis of chaperonin-dependent protein folding in Escherichia coli. Cell 122, 209-220 (2005).

58. Wilhelm, M. et al. Mass-spectrometry-based draft of the human proteome. Nature 509, 582-587 (2014).

59. Giessen, T. W. \& Silver, P. A. Widespread distribution of encapsulin nanocompartments reveals functional diversity. Nat. Microbiol. 2, 17029 (2017).

60. Matsumoto, Y. \& Jasanoff, A. T2 relaxation induced by clusters of superparamagnetic nanoparticles: Monte Carlo simulations. Magn. Reson. Imaging 26, 994-998 (2008). 
61. Giessen, T. W. Encapsulins: microbial nanocompartments with applications in biomedicine, nanobiotechnology and materials science. Curr. Opin. Chem. Biol. 34, 1-10 (2016).

62. Meister, M. Physical limits to magnetogenetics. Elife 5, e17210 (2016).

63. Wang, Q., Mercogliano, C. P. \& Löwe, J. A ferritin-based label for cellular electron cryotomography. Structure 19, 147-154 (2011).

64. Matsumoto, Y., Chen, R., Anikeeva, P. \& Jasanoff, A. Engineering intracellular biomineralization and biosensing by a magnetic protein. Nat. Commun. 6 , 8721 (2015).

65. Risco, C. et al. Specific, sensitive, high-resolution detection of protein molecules in eukaryotic cells using metal-tagging transmission electron microscopy. Structure 20, 759-766 (2012).

66. Shu, X. et al. A genetically encoded tag for correlated light and electron microscopy of intact cells, tissues, and organisms. PLoS Biol. 9, e1001041 (2011).

67. Joesch, M. et al. Reconstruction of genetically identified neurons imaged by serial-section electron microscopy. Elife 5, e15015 (2016).

68. Martell, J. D. et al. Engineered ascorbate peroxidase as a genetically encoded reporter for electron microscopy. Nat. Biotechnol. 30, 1143-1148 (2012).

69. Wickersham, I. R., Finke, S., Conzelmann, K.-K. \& Callaway, E. M. Retrograde neuronal tracing with a deletion-mutant rabies virus. Nat. Methods 4, 47-49 (2007).

70. Chang, Y.-W. et al. Correlated cryogenic photoactivated localization microscopy and cryo-electron tomography. Nat. Methods 11, 737-739 (2014).

71. van den Heuvel, R. H. H. et al. Improving the performance of a quadrupole time-of-flight instrument for macromolecular mass spectrometry. Anal. Chem. 78, 7473-7483 (2006)

72. Lorenzen, K., Versluis, C., van Duijn, E., van den Heuvel, R. H. H. \& Heck, A. J. R. Optimizing macromolecular tandem mass spectrometry of large noncovalent complexes using heavy collision gases. Int. J. Mass. Spectrom. 268, 198-206 (2007).

73. Snijder, J., Rose, R. J., Veesler, D., Johnson, J. E. \& Heck, A. J. R. Studying 18 $\mathrm{MDa}$ virus assemblies with native mass spectrometry. Angew. Chem. Int. Ed. Engl. 52, 4020-4023 (2013).

74. Veesler, D. et al. Architecture of a dsDNA viral capsid in complex with its maturation protease. Structure 22, 230-237 (2014).

75. Desai, M., Slusarczyk, A. L., Chapin, A., Barch, M. \& Jasanoff, A. Molecular imaging with engineered physiology. Nat. Commun. 7, 13607 (2016).

\section{Acknowledgements}

We are grateful for support from the European Research Council under grant agreements ERC-StG: 311552 (F.S., A.S., H.R., G.G.W.). T.W., J.S. and A.J.R.H acknowledge support from the Netherlands Organization for Scientific Research (NWO) funding the largescale proteomics facility Proteins@Work (project 184.032.201) embedded in the Netherlands Proteomics Centre. We acknowledge Susanne Pettinger for assistance with DLS measurements.

\section{Author contributions}

F.S. co-designed the study, generated all constructs, conducted all cell and biochemical experiments, analyzed data, generated figures and co-wrote the manuscript; C.M. made important contributions to the iron-loading experiments and performed in vitro MRI experiments; P.E. designed, conducted and analyzed cryo-EM experiments supervised by J.P.; A.S. supported cell experiments; H.R. supported animal experiments; M.D. and S.B. conducted in vivo MRI experiments supervised by A.J.; A.G. helped with HPLC purification and DLS analysis; T.P.W. and J.S. conducted and analyzed native mass spectrometry experiments supervised by A.J.R.H., H.F. and M.H. supervised in vitro MRI experiments; V.N. supervised MSOT experiments; G.G.W. conceptualized and codesigned the study, analyzed data and generated figures, supervised the project, and wrote the manuscript.

\section{Additional information}

Supplementary Information accompanies this paper at https://doi.org/10.1038/s41467018-04227-3.

Competing interests: The authors declare no competing interests.

Reprints and permission information is available online at http://npg.nature.com/ reprintsandpermissions/

Publisher's note: Springer Nature remains neutral with regard to jurisdictional claims in published maps and institutional affiliations.

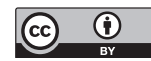

Open Access This article is licensed under a Creative Commons Attribution 4.0 International License, which permits use, sharing, adaptation, distribution and reproduction in any medium or format, as long as you give appropriate credit to the original author(s) and the source, provide a link to the Creative Commons license, and indicate if changes were made. The images or other third party material in this article are included in the article's Creative Commons license, unless indicated otherwise in a credit line to the material. If material is not included in the article's Creative Commons license and your intended use is not permitted by statutory regulation or exceeds the permitted use, you will need to obtain permission directly from the copyright holder. To view a copy of this license, visit http://creativecommons.org/ licenses/by/4.0/.

(C) The Author(s) 2018 